This is an Open Access article, distributed under the terms of the Creative Commons Attribution licence (http://creativecommons.org/licenses/by/4.0/), which permits unrestricted re-use, distribution, and reproduction in any medium, provided the original work is properly cited.

\title{
Recent progress in astrophysical plasma turbulence from solar wind observations
}

\author{
C. H. K. Chen $\dagger$ \\ Department of Physics, Imperial College London, London SW7 2AZ, UK \\ (Received 7 September 2016; revised 8 November 2016; accepted 11 November 2016)
}

This paper summarises some of the recent progress that has been made in understanding astrophysical plasma turbulence in the solar wind, from in situ spacecraft observations. At large scales, where the turbulence is predominantly Alfvénic, measurements of critical balance, residual energy and three-dimensional structure are discussed, along with comparison to recent models of strong Alfvénic turbulence. At these scales, a few per cent of the energy is also in compressive fluctuations, and their nature, anisotropy and relation to the Alfvénic component is described. In the small-scale kinetic range, below the ion gyroscale, the turbulence becomes predominantly kinetic Alfvén in nature, and measurements of the spectra, anisotropy and intermittency of this turbulence are discussed with respect to recent cascade models. One of the major remaining questions is how the turbulent energy is dissipated, and some recent work on this question, in addition to future space missions which will help to answer it, are briefly discussed.

Key words: astrophysical plasmas, plasma nonlinear phenomena, space plasma physics

\section{Introduction}

Plasma turbulence is one of the most widespread collective phenomena occurring in nature. It appears to be present throughout the universe, in a diverse range of environments, including galaxy clusters, accretion disks, the interstellar medium, stars, stellar winds and planetary magnetospheres. While being an intriguing and complex aspect of our universe of intrinsic interest, it can also have an important impact on the large-scale properties of these systems. For example, it can enable angular momentum transport in accretion disks (Balbus \& Hawley 1998), galactic magnetic field amplification (Kulsrud \& Zweibel 2008), limit thermal conduction in galaxy clusters (Schekochihin et al. 2008), determine the dispersal and mixing of elements in the interstellar medium (Scalo \& Elmegreen 2004) and play a key role in star formation (McKee \& Ostriker 2007). In addition, the dissipation of turbulent energy may explain the large temperatures observed in many astrophysical systems, such as the solar corona (Cranmer et al. 2015) and galaxy clusters (Zhuravleva et al. 2014). Turbulent plasmas display complex, chaotic, broadband fluctuations, which

$\dagger$ Email address for correspondence: christopher.chen@imperial.ac.uk 
are generally interpreted as a scale-invariant cascade of energy from large scales, where the energy is injected, to small scales, where it is dissipated. However, many questions remain about both the cascade and dissipation processes.

The solar wind presents one of the best places to understand the basic physics of plasma turbulence. The main reason for this is that in situ spacecraft measurements are possible, meaning that a wealth of information is available, unlike for plasmas outside our solar system. It is also fast flowing, travelling much quicker than the local Alfvén speed and turbulent fluctuation amplitudes, meaning that time series measured by spacecraft can generally be interpreted as spatial cuts though the plasma (the Taylor hypothesis), enabling them to be readily compared to theoretical predictions. The majority of solar wind turbulence measurements, and most of those described in this paper, have been made in the near-Earth solar wind. Typical plasma conditions here $^{1}$ are: magnetic field strength $B \sim 6 \mathrm{nT}$, number density $n_{i} \sim n_{e} \sim 11 \mathrm{~cm}^{-3}$, bulk speed $v_{i} \sim v_{e} \sim 390 \mathrm{~km} \mathrm{~s}^{-1}$ and ion and electron temperatures $T_{i} \sim 6 \mathrm{eV}$ and $T_{e} \sim 12 \mathrm{eV}$, which give ion and electron plasma betas of order unity, $\beta_{i} \sim \beta_{e} \sim 1$. The dominant ion species (to which these parameters refer) is hydrogen, with helium and other minor ions making up a few per cent of the solar wind by number density. It is important to note, however, that there is large a variation in all of these values, making the solar wind an ideal place for parameter studies of plasma processes, applicable to a wide variety of astrophysical environments.

This paper summarises some of the recent progress that has been made in understanding solar wind turbulence. It is not intended to be a complete review; for general review papers on solar wind observations see, e.g. Alexandrova et al. (2013), Bruno \& Carbone (2013) and the collection of Kiyani, Osman \& Chapman (2015). It also primarily summarises work in which I have been involved, with other results and theoretical background described for context. Therefore, it is not an unbiased review, but presents a viewpoint based on the latest observations and theoretical developments.

\section{Alfvénic turbulence}

The solar wind has long been known to contain large-scale Alfvénically polarised fluctuations, consistent with predominant propagation away from the Sun (e.g. Belcher \& Davis 1971). These are thought to be Alfvén waves, generated at or near the Sun, which propagate into interplanetary space and drive a turbulent cascade. The magnetic fluctuations at these large scales are often seen to have a $1 / f$ frequency spectrum, which has been interpreted as arising from uncorrelated processes at the Sun (Matthaeus \& Goldstein 1986) or a reflection-driven cascade (Velli, Grappin \& Mangeney 1989; Verdini et al. 2012; Perez \& Chandran 2013). Here, we are concerned with the smaller-scale turbulence driven by these large-scale fluctuations. In this range, the Alfvénically polarised fluctuations remain dominant, and in this section our recent progress on this turbulence is discussed.

\subsection{Phenomenological models of Alfvénic turbulence}

Alfvénic turbulence is thought to be captured by the incompressible magnetohydrodynamics (MHD) equations, which in fluctuating Elsasser (1950) form (omitting forcing and dissipation terms) are

$$
\begin{gathered}
\frac{\partial \delta z^{ \pm}}{\partial t} \mp v_{A} \nabla_{\|} \delta z^{ \pm}+\delta z^{\mp} \cdot \nabla \delta z^{ \pm}=-\nabla P, \\
\nabla \cdot \delta z^{ \pm}=0,
\end{gathered}
$$

${ }^{1}$ As measured by the Wind spacecraft using the data set described in Chen et al. (2016). 
where $\delta \boldsymbol{z}^{ \pm}=\delta \boldsymbol{v} \pm \delta \boldsymbol{b}$ are the fluctuating Elsasser variables, $\boldsymbol{v}$ is the bulk velocity, $\boldsymbol{b}=\boldsymbol{B} / \sqrt{\mu_{0} \rho}$ is the magnetic field in Alfvén units, $\rho$ is the mass density, $v_{A}=$ $B_{0} / \sqrt{\mu_{0} \rho}$ is the Alfvén speed, $P$ contains the total (thermal plus magnetic) pressure and $\nabla_{\|}$is the gradient in the direction of the mean magnetic field $\boldsymbol{B}_{0}$. An early model of Alfvénic turbulence, based on these equations, was developed by Iroshnikov (1963) and Kraichnan (1965). From the form of the nonlinear terms $\left(\delta z^{\mp} \cdot \nabla \delta z^{ \pm}\right)$ it was realised that nonlinear interactions occur via oppositely propagating Alfvén wave packets $\left(\delta z^{+}\right.$and $\left.\delta z^{-}\right)$. The turbulence was assumed to be isotropic so that the presence of a strong mean magnetic field would lead to weak interactions (the linear terms dominating in (2.1)), requiring many such interactions to transfer the energy to a smaller scale. When a constant energy flux through scales is assumed, scaling arguments based on the cascade time lead to an inertial range total energy spectrum

$$
E(k) \propto k^{-3 / 2},
$$

where $k$ is the wavenumber of the fluctuations.

Weak Alfvénic turbulence, however, was later shown to develop strong anisotropy, with a spectrum perpendicular to mean magnetic field of $E\left(k_{\perp}\right) \propto k_{\perp}^{-2}$ and no transfer in the parallel direction (Montgomery \& Turner 1981; Shebalin, Matthaeus \& Montgomery 1983; Goldreich \& Sridhar 1997; Galtier et al. 2000). This leads to a violation of both the isotropic and weak assumptions as the cascade proceeds to smaller scales, since the degree of nonlinearity increases with wavenumber. Goldreich \& Sridhar (1995) proposed that a state of critical balance is reached in which the linear and nonlinear terms become (and remain) comparable, and the transfer to smaller scales occurs within a single interaction. From (2.1), the linear Alfvén time is $\tau_{A} \sim l_{\|} / v_{A}$ (where $l_{\|}$is the scale parallel to the mean magnetic field) and the nonlinear time was estimated as $\tau_{n l} \sim l_{\perp} / \delta z$ (where $l_{\perp}$ is the perpendicular scale), which, when equated, lead to a scaling $k_{\|} \propto k_{\perp}^{2 / 3} 2$. This results in an anisotropy $k_{\perp} \gg k_{\|}$at small scales and energy spectra

$$
E\left(k_{\perp}\right) \propto k_{\perp}^{-5 / 3}, \quad E\left(k_{\|}\right) \propto k_{\|}^{-2} .
$$

Since the nonlinear transfer occurs within one interaction, turbulence in critical balance is considered to be strong at all scales, even when $\delta B / B_{0} \ll 1$.

Boldyrev (2006) later noted that the nonlinear time should contain an extra factor of the alignment angle $\theta$ between the fluctuations $\tau_{n l} \sim l_{\perp} /(\delta z \sin \theta)$. This is because both the fluctuations and their gradients are mostly in the plane perpendicular to the mean field, and within this plane the gradients are perpendicular to the fluctuations due to solenoidality, leading to a greater reduction of the nonlinear terms if the fluctuations are more aligned. It was proposed that $\delta \boldsymbol{v}$ and $\delta \boldsymbol{b}$ align to the maximum amount permitted at each scale, leading to $\theta \propto k_{\perp}^{-1 / 4}$, and three-dimensionally anisotropic eddies at small scales $l_{\|} \gg \xi \gg \lambda$, where $\xi$ is the characteristic length in the fluctuation direction and $\lambda$ in the direction perpendicular to $l_{\|}$and $\xi$. The resulting spectra are

$$
E\left(k_{\lambda}\right) \propto k_{\lambda}^{-3 / 2}, \quad E\left(k_{\xi}\right) \propto k_{\xi}^{-5 / 3}, \quad E\left(k_{\|}\right) \propto k_{\|}^{-2} .
$$

In more recent years, further additions to these models have been proposed to allow for the imbalance of the oppositely directed Alfvénic fluxes (Lithwick, Goldreich \& Sridhar 2007; Beresnyak \& Lazarian 2008; Chandran 2008; Perez \& Boldyrev

\footnotetext{
${ }^{2}$ This relation, and that fact that it leads to increasing anisotropy towards smaller scales, was also noted in the previous work of Higdon (1984).
} 
2009; Podesta \& Bhattacharjee 2010) and intermittency (Chandran, Schekochihin \& Mallet 2015; Mallet \& Schekochihin 2016). While models such as these are at the phenomenological level, they provide scaling predictions that can be compared to observations to distinguish the physical processes taking place.

\subsection{Anisotropy and critical balance}

It is well known that Alfvénic turbulence in the solar wind is anisotropic (see reviews by Horbury, Wicks \& Chen 2012; Oughton et al. 2015). Correlation lengths are typically observed to be much longer in the direction parallel to the mean magnetic field than perpendicular ${ }^{3}$, consistent with the anisotropy $k_{\perp} \gg k_{\|}$implied by the above strong turbulence models. More recently, however, new techniques have been developed to measure the anisotropic scaling, as a more direct test. In the above models, the parallel length scale is associated with the linear term, corresponding to the propagation of Alfvén wave packets, which are sensitive to the mean magnetic field at their location and scale, i.e. the local mean field. The need to use such a mean field to test the critical balance predictions was identified in simulations (Cho \& Vishniac 2000; Maron \& Goldreich 2001) and a technique to do this in the solar wind, making use of the wavelet transform, was developed by Horbury, Forman \& Oughton (2008). At each location, and at each scale (i.e. for each wavelet coefficient in the transform), a local mean field can be defined from the average of the magnetic field weighted by the corresponding wavelet envelope. Fluctuations at a range of scales at a particular angle to the local mean field can be gathered to produce the spectrum in that direction ${ }^{4}$. A similar technique can be applied to structure functions (e.g. Luo \& Wu 2010; Chen et al. 2011b), for which the local mean field can be defined as the average between the points of the structure function. In practice, the results are not sensitive to this precise definition, as long as a local (and not global) mean field is used.

The results of the local structure function technique (Chen et al. 2011b), applied to the same magnetic field data as in Horbury et al. (2008), are shown in figure $1^{5}$. It can be seen that at large scales $\left(k \rho_{i} \lesssim 10^{-3}\right.$, where $\rho_{i}$ is the ion gyroradius) the turbulence is isotropic (Wicks et al. 2010) with $\delta B / B_{0} \sim 1$, and in the inertial range $\left(10^{-3} \lesssim k \rho_{i} \lesssim 1\right)$ the parallel spectrum is steeper than the perpendicular spectrum. The inertial range spectral index as a function of angle to the local mean field, $\theta_{B}$, is shown in figure $1(b)$. The perpendicular spectrum is closer to the $k_{\perp}^{-5 / 3}$ prediction of Goldreich \& Sridhar (1995) than the $k_{\perp}^{-3 / 2}$ prediction of Boldyrev (2006), but the parallel spectrum is consistent with the $k_{\|}^{-2}$ critical balance prediction of both models ${ }^{6}$. Wicks et al. (2011) also found a $k_{\|}^{-2}$ spectrum for the velocity and dominant Elsasser field (although instrument noise made it difficult to measure the anisotropy of the sub-dominant Elsasser field). It is important to note that the $k_{\|}^{-2}$ spectrum is only observed when a scale-dependent local mean field is used; a global mean field cannot be used to test critical balance, since, even for arbitrarily small $\delta B / B_{0}$, the predicted

\footnotetext{
${ }^{3}$ Although a notable exception is Dasso et al. (2005), who found the opposite situation in the fast solar wind at the large-scale end of the inertial range.

${ }^{4}$ Such conditioning means that higher (than second) order correlations are important for the local fluctuation spectrum (as noted by Matthaeus et al. 2012).

${ }^{5}$ The error bars here represent $(a)$ the measured statistical uncertainties on the structure functions, $(b)$ the measured uncertainties on the fitted spectral slopes and $(c)$ the uncertainties from the measured structure functions propagated to $\chi$.

${ }^{6} \mathrm{~A}$ recent suggestion by Beresnyak (2015) is that the $k_{\|}^{-2}$ spectrum could also be interpreted as a reflection of the Lagrangian frequency spectrum.
} 
(a)

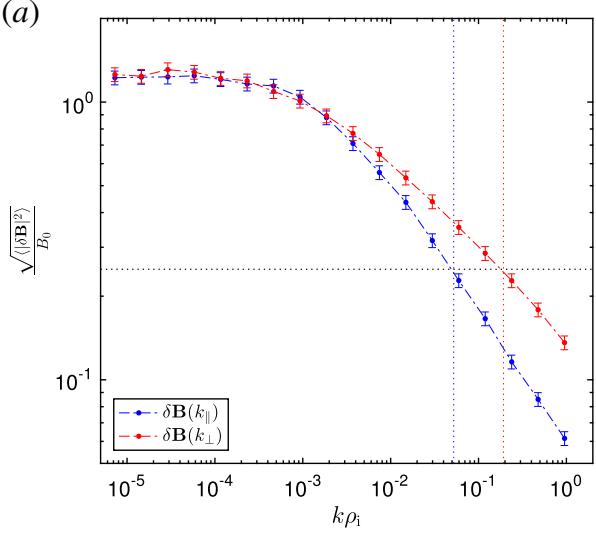

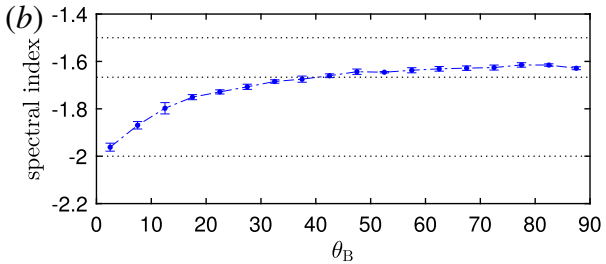

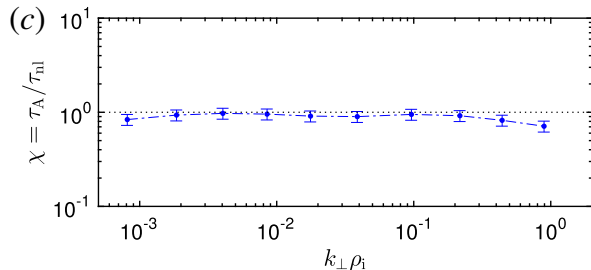

FIGURE 1. (a) Normalised magnetic fluctuation amplitude as a function of parallel and perpendicular wavenumber. (b) Variation of spectral index with angle to the local mean field $\theta_{B}$. (c) Ratio of linear and nonlinear time scales $\chi$ as a function of scale $k_{\perp} \rho_{i}$.

anisotropy would be just large enough that the true local parallel correlation would not be measured (Cho \& Vishniac 2000; Chen et al. 2011b).

An alternative way to test the critical balance condition is to estimate the turbulence strength directly from the measured time scales. The parallel and perpendicular lengths of an 'eddy' of amplitude $\delta B$ can be found using the technique of Chen et al. (2010b, $2012 a$ ). For a particular $\delta B$, for example the horizontal dotted line in figure $1(a), k_{\|}$ is estimated as the wavenumber corresponding to the parallel spectrum (blue vertical dotted line) and $k_{\perp}$ as the wavenumber corresponding to the perpendicular spectrum (red vertical dotted line). From this, the wavevector anisotropy can be determined, e.g. at $k_{\perp} \rho_{i}=1$ the anisotropy is $k_{\perp} / k_{\|}=5.5 \pm 0.7$, corresponding to a wavevector angle $\theta_{k B}=(79.7 \pm 1.2)^{\circ}$. The ratio of linear to nonlinear times (omitting the alignment angle) is then given by $\chi=\tau_{A} / \tau_{n l}=\left(k_{\perp} / k_{\|}\right)\left(\delta B / B_{0}\right)$ and is shown in figure $1(c)$ as a function of $k_{\perp} \rho_{i}$, where it can be seen to be close to unity throughout the inertial range $^{7}$. Even though the estimate of $\tau_{n l}$ used here is based on the magnetic fluctuations only, does not include the alignment angle and is only defined to order unity, the critical balance condition $(\chi \sim 1)$ appears to be well satisfied in the solar wind.

\subsection{Spectral indices and residual energy}

While the results of the previous section would appear to favour the Goldreich \& Sridhar (1995) model of Alfvénic turbulence, the situation becomes more complicated when also considering the velocity and electric field. The electric field was measured by Bale et al. (2005) to follow the magnetic field, having a spectral index of $-5 / 3$ at MHD scales ${ }^{8}$. Since the perpendicular plasma velocity in this range should be the $E \times B$ drift, the velocity was expected to display a similar spectrum, however, it has been shown to be shallower, with a value nearer $-3 / 2$ (e.g. Mangeney et al. 2001; Podesta, Roberts \& Goldstein 2007). How can these spectra be consistent with

\footnotetext{
${ }^{7}$ Note that this is different to the result reported by Wang et al. (2016), however, in that study a fixed scale-independent anisotropy was assumed in the calculation of $\chi$.

${ }^{8}$ Note that because of the anisotropy $k_{\perp} \gg k_{\|}$, spectra measured in the solar wind generally correspond to $k_{\perp}$ spectra (unless the local mean field technique of $\S 2.2$ is used).
} 

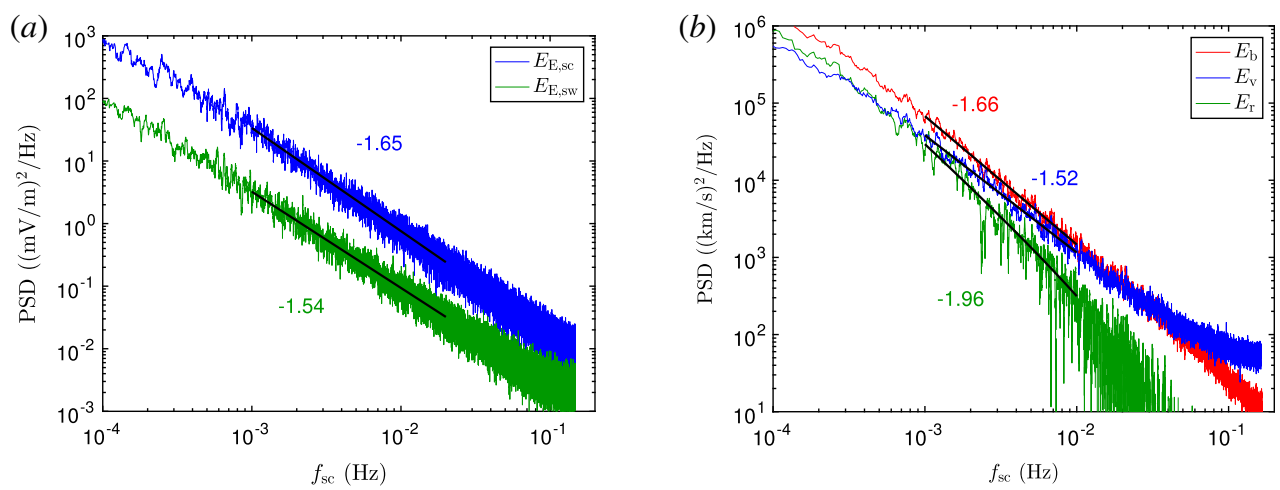

FIGURE 2. (a) Electric field spectrum in the spacecraft frame (blue) and plasma frame (green) (adapted from Chen et al. 2011a). (b) Magnetic (red), velocity (blue) and residual energy (green) spectra (adapted from Chen et al. 2013a). Note that the flattening of $E_{v}$ and steepening of $E_{r}$ for $f_{s c}>10^{-2} \mathrm{~Hz}$ are artificial (due to instrument noise).

each other? The answer lies in the frame dependence of the electric field (Chen et al. 2011a). Figure 2 shows the electric field spectrum measured in the spacecraft frame $\boldsymbol{E}_{s c}$ with a spectral index near $-5 / 3$, similar to the magnetic field. It also shows the electric field spectrum after Lorentz transforming to the frame of the mean solar wind velocity $\boldsymbol{v}_{0}$,

$$
\boldsymbol{E}_{s w}=\boldsymbol{E}_{s c}+\boldsymbol{v}_{0} \times \boldsymbol{B} .
$$

It can be seen that the solar wind frame spectrum is lower by an order of magnitude and is shallower, having a spectral index close to $-3 / 2$. These spectra are, therefore, in fact consistent with the velocity and magnetic field measurements, and are independent confirmation of the difference between them. The $\delta \boldsymbol{E}_{s w}$ spectrum matches the velocity spectrum since to leading order it is given by $\delta \boldsymbol{E}_{s w}=-\delta \boldsymbol{v} \times \boldsymbol{B}_{0}$, and the $\delta \boldsymbol{E}_{s c}$ spectrum matches the magnetic spectrum since to leading order it is $\delta \boldsymbol{E}_{s c}=-\boldsymbol{v}_{0} \times \delta \boldsymbol{B}$, i.e. it is dominated by the magnetic fluctuations convected at the mean solar wind velocity.

While the electric, magnetic and velocity spectra are self-consistent, the question remains as to why the velocity and magnetic fluctuations differ. This difference is known as the residual energy, and its spectrum is defined as $E_{r}(\boldsymbol{k})=E_{v}(\boldsymbol{k})-E_{b}(\boldsymbol{k})$. A measure of the amount of residual energy is the Alfvén ratio $r_{A}=\delta \boldsymbol{v}^{2} / \delta \boldsymbol{b}^{2}$, which is $r_{A}=1$ for a pure Alfvén wave, but is measured to be $r_{A} \approx 0.7$ in the solar wind (Chen et al. 2013a). Statistical arguments have been made to explain this dominance of magnetic fluctuation energy (e.g. Frisch et al. 1975; Pouquet, Frisch \& Leorat 1976; Boldyrev, Perez \& Zhdankin 2012b). The existence of residual energy, both theoretically and observationally, indicates that strong turbulence can produce quantitative differences to the linear wave relationships.

The residual energy has also been described as a balance between the Alfvén effect (linear term) leading to equipartition and a turbulent dynamo (nonlinear term) generating the magnetic excess (Grappin, Leorat \& Pouquet 1983; Müller \& Grappin 2005). Müller \& Grappin (2005) used an isotropic closure theory to suggest that $E_{r}$ varies with the total energy spectrum $E_{t}$, following $k^{-2}$ for a $k^{-3 / 2}$ total energy spectrum and $k^{-7 / 3}$ for a $k^{-5 / 3}$ total energy spectrum. Boldyrev, Perez \& Wang (2012a) extended this to an anisotropic model in which the perpendicular spectra 
(a)

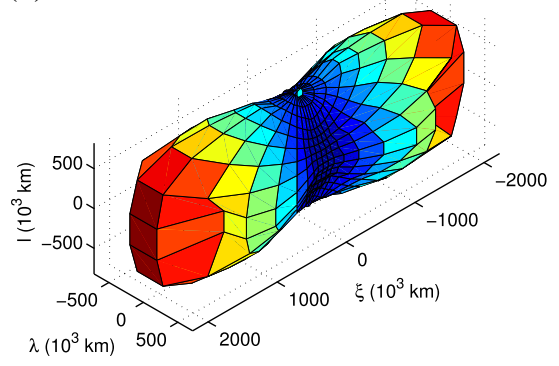

(b)

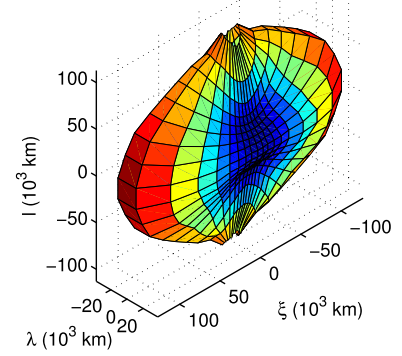

(c)

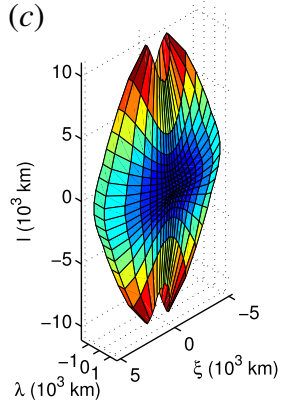

FIgURE 3. Three-dimensional magnetic eddy shapes from large $(a)$ to small $(c)$ scales, in which $l$ is in the local mean field direction, $\xi$ the local $\delta \boldsymbol{B}_{\perp}$ direction, $\lambda$ perpendicular to these and colour represents distance from the origin (from Chen et al. 2012a).

are $E_{r}\left(k_{\perp}\right) \propto k_{\perp}^{-1}$ for weak and $E_{r}\left(k_{\perp}\right) \propto k_{\perp}^{-2}$ for strong turbulence; in both cases, $E_{r}\left(k_{\|}, k_{\perp}\right)$ is concentrated near the $k_{\perp}$ axis, similar to the total energy. In the solar wind, the residual energy has an average spectral index of $-1.9^{9}$ (Chen et al. 2013a), and an example spectrum is shown in figure 2, along with the velocity and magnetic spectra $^{10}$. There is also evidence that it is anisotropic and concentrated in the region near $k_{\|} \approx 0$ (Yan et al. 2016). These observations would be most consistent with the strong turbulence model of Boldyrev et al. (2012a), which provides a possible explanation for the different scaling of the velocity and magnetic fluctuations.

\subsection{Three-dimensional anisotropy}

Alfvénic turbulence is polarised such that magnetic fluctuations are in the plane perpendicular to the mean field, i.e. $\delta B_{\perp} \gg \delta B_{\|}$. The local $\delta \boldsymbol{B}_{\perp}$ direction within this perpendicular plane breaks the symmetry about the mean field, meaning that the turbulence can be three-dimensionally anisotropic, e.g. as in the Boldyrev (2006) model (\$2.1). The techniques of $\$ 2.2$ were recently extended to measure this three-dimensional (3-D) anisotropy in the solar wind (Chen et al. 2012a). As well as the local mean field, a local $\delta \boldsymbol{B}$ direction can be defined and a 3-D coordinate system constructed, with one axis in the local mean field direction, one in the $\delta \boldsymbol{B}_{\perp}$ direction and the third perpendicular to these. By selecting different $\delta B$ values, and finding the corresponding lengths in each direction, Chen et al. (2012a) constructed the 3-D eddy shapes of the magnetic fluctuations at several scales, shown in figure 3. They vary from being extended in the $\delta \boldsymbol{B}_{\perp}$ direction at large scales, to being three-dimensionally anisotropic in the sense of Boldyrev (2006) at small scales. While the small-scale anisotropy $\left(l_{\|}>\xi>\lambda\right)$ and the scaling in the $l_{\|}$and $\xi$ directions are consistent with the Boldyrev (2006) model, the $\lambda$ scaling is not $^{11}$.

There are a few considerations when interpreting these results. Firstly, one might expect the anisotropy with respect to the $\delta \boldsymbol{B}$ direction to be a simple result of the

\footnotetext{
${ }^{9}$ While $E_{b}, E_{v}, E_{t}$ and $E_{r}$ cannot all be true power laws, it is not possible to tell which ones are from observations due to the limited scaling range. In the theoretical models, it is $E_{t}$ and $E_{r}$ which are the power laws, leading to a steeper $E_{b}$ and shallower $E_{v}$.

${ }^{10}$ The Alfvén unit normalisation for the magnetic field here uses the mean density $\rho_{0}$, which is appropriate for $\delta \rho / \rho_{0} \ll 1$, as is typically the case in the solar wind (see $\S 3$ ).

${ }^{11}$ Another test of this model is the scaling of the alignment angles, however, this is difficult to measure due to current instrument limitations (Podesta et al. 2009; Wicks et al. 2013).
} 
solenoidality of the magnetic field. However, it can be shown that solenoidality alone does not determine the anisotropy in the local perpendicular plane (Chen et al. 2012a; Mallet et al. 2016), and extra physics, such as alignment (Boldyrev 2006) is involved. Secondly, it has been suggested that the anisotropy in the perpendicular plane at large scales could be a result of the solar wind expansion as it travels away from the Sun. Verdini \& Grappin (2015) argued that magnetic flux conservation in the expanding wind causes fluctuations in the radial direction to be smaller, leading to a bias towards lower fluctuation amplitudes in the $\xi$ direction when measurements are made in the radial direction. Some evidence in support of this hypothesis has recently been reported (Vech \& Chen 2016). As well as the eddy shapes, this effect could also alter the 3-D scaling, since any effect of the expansion relative to the turbulent dynamics is likely to be scale dependent. Finally, as discussed in $\S 2.3$, the situation is further complicated by the presence of residual energy, which causes the magnetic spectrum to be steeper than the velocity spectrum; how this impacts the 3 -D anisotropy is not yet well understood. Therefore, while observations show that Alfvénic turbulence in the solar wind is locally three-dimensionally anisotropic, more work is required to fully understand this.

\subsection{Imbalanced turbulence}

As shown in $\S 2.3$, the scalings of the velocity and magnetic fluctuations are different, so it is the total energy spectrum $E_{t}(\boldsymbol{k})=E_{v}(\boldsymbol{k})+E_{b}(\boldsymbol{k})$ which should be compared to the models of $\S 2.1$. These models, however, describe balanced Alfvénic turbulence, i.e. turbulence with equal fluxes of Alfvénic fluctuations propagating in each direction along the mean field. In real systems, turbulence is often imbalanced, with unequal fluxes created by localised driving sources, such as supernovae in the interstellar medium or Alfvén waves from the Sun. In the solar wind, the level of imbalance can be quantified with the normalised cross-helicity $\sigma_{c}=2\langle\delta \boldsymbol{v} \cdot \delta \boldsymbol{b}\rangle /\left\langle\delta \boldsymbol{v}^{2}+\delta \boldsymbol{b}^{2}\right\rangle ; \sigma_{c} \approx 0$ corresponds to balanced turbulence and $\sigma_{c} \approx \pm 1$ to highly imbalanced turbulence. While the solar wind at $1 \mathrm{AU}$ has a typical imbalance of $\sigma_{c}=0.46$ (Chen et al. 2013a), there is significant variability, enabling the systematic dependence to be studied.

Figure 4 shows the dependence of the spectral indices on the level of imbalance (all quantities are measured in the middle of the inertial range). The total energy spectral index varies from $\approx-5 / 3$ at low imbalance to $\approx-3 / 2$ at high imbalance (as also found by Podesta \& Borovsky 2010). $E_{b}$ and $E_{v}$ scale similarly for $\left|\sigma_{c}\right| \approx 1$, which is consistent with the residual energy being mathematically constrained to be small for large imbalance. It is interesting, however, that they take a value close to $-3 / 2$, favouring the Boldyrev (2006) model in the absence of residual energy. The residual energy itself has a spectral index of -2 (see § 2.3) for low imbalance, which becomes shallower for larger imbalance ${ }^{12}$. Both Elsasser variables (not shown here) have the same scaling as the total energy, to within uncertainties, for all $\left|\sigma_{c}\right|^{13}$. The main dependence of the total energy spectrum with $\sigma_{c}$, however, is not predicted by any of the current models of imbalanced Alfvénic turbulence (e.g. Lithwick et al. 2007; Beresnyak \& Lazarian 2008; Chandran 2008; Perez \& Boldyrev 2009; Podesta $\&$ Bhattacharjee 2010). Therefore, while several aspects of Alfvénic turbulence in the solar wind are beginning to be understood, a complete explanation still remains to be found.

\footnotetext{
${ }^{12}$ Although as noted by Chen et al. (2013a), because the amount of residual energy is small at large imbalance, there may be a systematic bias towards shallower spectra here due to small inaccuracies in the magnetic field normalisation.

${ }^{13}$ This is true except for the two largest $\left|\sigma_{c}\right|$ bins where the $z^{-}$spectrum is affected by instrument noise.
} 


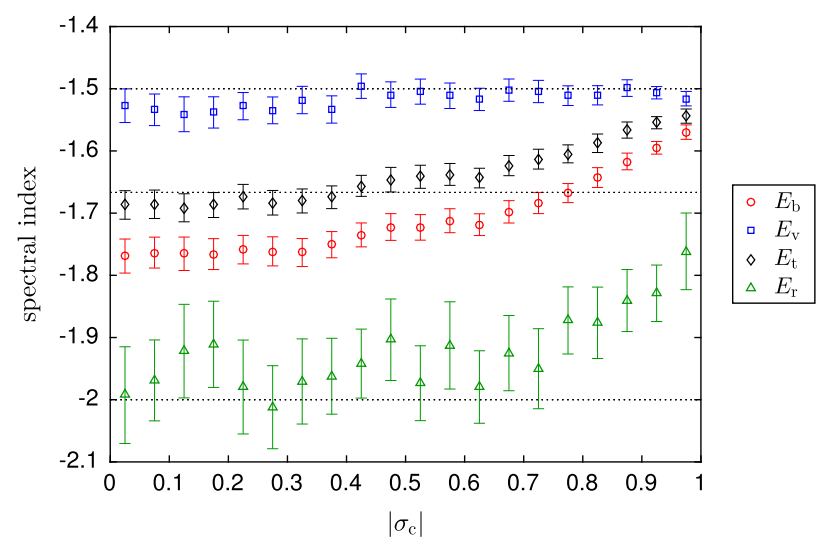

FIGURE 4. Variation of spectral indices of magnetic field $\left(E_{b}\right)$, velocity $\left(E_{v}\right)$, total energy $\left(E_{t}\right)$ and residual energy $\left(E_{r}\right)$ with the level of imbalance $\left|\sigma_{c}\right|$ (adapted from Chen et al. $2013 a)$.

\section{Compressive fluctuations}

While the dominant power in the solar wind is in the Alfvénically polarised fluctuations, there is also a measurable fraction in non-Alfvénic modes, involving variations of the density $\delta n$ and magnetic field magnitude $\delta|\boldsymbol{B}|$. As well as being of intrinsic interest, understanding this compressive component is particularly important for interpreting measurements of plasmas outside our solar system, where density fluctuations are usually the most observationally accessible quantity, for example in the interstellar medium (Armstrong, Rickett \& Spangler 1995) and galaxy clusters (Zhuravleva et al. 2014). They are also thought to play a key role in the star formation process (McKee \& Ostriker 2007). The distribution of the magnetic compressibility in the solar wind at the $1 \mathrm{~h}$ scale $\left(k \rho_{i} \sim 10^{-3}\right)$ is shown in figure 5; the average fraction of magnetic energy in the compressive component is $\sim 2 \%{ }^{14}$. What is the nature of this component and how does it interact with the Alfvénic turbulence? In this section, our recent progress on these questions is discussed.

\subsection{Spectra and passivity}

It was proposed by Higdon (1984) that the density variations observed in interstellar turbulence are entropy fluctuations, and possibly magnetosonic waves, passively mixed by the Alfvénic turbulence. This concept was developed further by Goldreich \& Sridhar (1997), who suggested that for MHD turbulence, slow waves (or pseudo-Alfvén waves in the incompressible limit) would be passive to the Alfvénic turbulence as a result of the anisotropy $k_{\perp} \gg k_{\|}$developed by the cascade. An Alfvén wave $\left(\delta \boldsymbol{z}_{A}^{ \pm}\right)$is polarised perpendicular to $\boldsymbol{B}_{0}$, and distorts a counter-propagating Alfvén wave packet through the term $\delta z_{A}^{ \pm} \cdot \nabla \delta z_{A}^{\mp}$, which is of order $\sim k_{\perp} \delta z_{A}^{2}$. However, an oblique slow wave $\left(\delta \boldsymbol{z}_{s}^{ \pm}\right)$is polarised mostly parallel to $\boldsymbol{B}_{0}$, so distorts a counter-propagating Alfvén wave packet through the term $\delta \boldsymbol{z}_{s}^{ \pm} \cdot \nabla \delta \boldsymbol{z}_{A}^{\mp}$, which is of order $\sim \delta z_{s} k_{\|} \delta z_{A}$, i.e. a factor of $k_{\|} / k_{\perp}$ smaller (assuming equal amplitudes). This would mean that slow wave fluctuations are scattered by the anisotropic Alfvénic turbulence, but do not actively interfere with it, which would also result in negligible

\footnotetext{
${ }^{14}$ When using $\delta B_{\|}$instead of $\delta|\boldsymbol{B}|$ to define the magnetic compressibility this value is $\sim 10 \%$.
} 

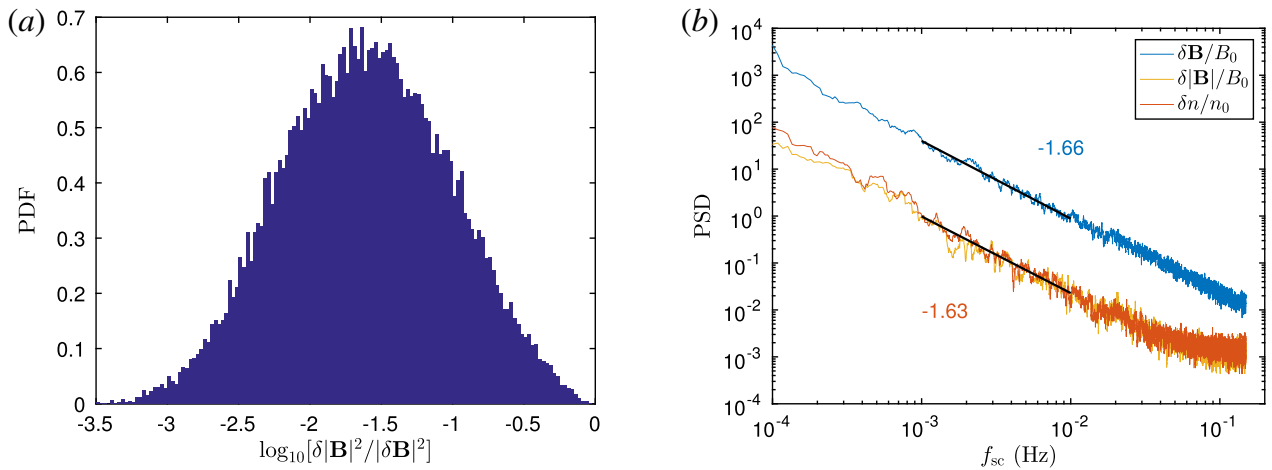

FIgURE 5. (a) Distribution of magnetic compressibility at the outer scale of the Alfvénic inertial range. (b) Normalised spectra of compressive fluctuations, $\delta n$ and $\delta|\boldsymbol{B}|$, in comparison to the total magnetic fluctuation spectrum, $\delta \boldsymbol{B}$.

energy transfer from the Alfvénic to slow mode component (Goldreich \& Sridhar 1995; Maron \& Goldreich 2001). Schekochihin et al. (2009) argued that for weakly collisional plasmas such as the solar wind, the MHD description is not sufficient for the compressive fluctuations ${ }^{15}$, and a kinetic treatment is required. In the gyrokinetic formalism, where $k_{\perp} \gg k_{\|}$is taken as an ordering assumption, the nonlinear fluid equations for the Alfvénic turbulence at $k_{\perp} \rho_{i} \ll 1$ (Reduced MHD) decouple from the ion kinetic equation. The active Alfvénic turbulence is self-contained, and passively mixes the non-Alfvénic part of the ion distribution, from which the compressive fluctuations (which produce the kinetic counterparts of the slow and entropy modes) are obtained (Schekochihin et al. 2009). Therefore, the passivity of these modes, originally derived in the context of fluid models, is predicted to hold for kinetic plasmas too.

In hydrodynamic turbulence, a passive scalar $\sigma$ shares the same spectrum as the advecting velocity field $\boldsymbol{v}$ (neglecting intermittency). This is because it follows its continuity equation, $\partial \sigma / \partial t+\boldsymbol{v} \cdot \nabla \sigma=0$, meaning that its nonlinear time $\sim l / \delta v$ is the same as that of the active turbulence, leading to $\sigma \propto \delta v$. In plasma turbulence, however, the situation is more complicated, since the nonlinear times involve both the velocity and magnetic fields, and their alignment angles (see $§ 2.1$ ). It is therefore of interest to compare the scaling of the compressive fluctuations in the solar wind to that of the Alfvénic turbulence. While spectra of compressive fluctuations have long been measured in the solar wind (see reviews by Alexandrova et al. 2013; Bruno \& Carbone 2013), we have recently been able to investigate these with greater precision, to enable comparison to the Alfvénic turbulence. Chen et al. (2011a) showed that the spectral indices of both $\delta n$ and $\delta|\boldsymbol{B}|$ are close to $-5 / 3$, similar to the magnetic field, rather than the velocity, which has a $-3 / 2$ spectral index. An example from this data set is shown in figure 5, where the typical features can be seen: compressive fluctuations at a level much lower than the total power, with a spectral slope similar to that of the Alfvénic magnetic fluctuations. This scaling of the compressive fluctuations indicates that they are not passively advected in the hydrodynamic sense. It is still possible, however, that they are passive to the Alfvénic turbulence, in which they are mixed by both the velocity and magnetic fluctuations.

\footnotetext{
${ }^{15}$ Although for the Alfvénic component that it would be sufficient, since small amplitude $\left(\delta B / B_{0} \ll 1\right)$ Alfvén waves at $k_{\perp} \rho_{i} \ll 1$ do not modify the Maxwellian nature of the ion distribution (Schekochihin et al. 2009).
} 


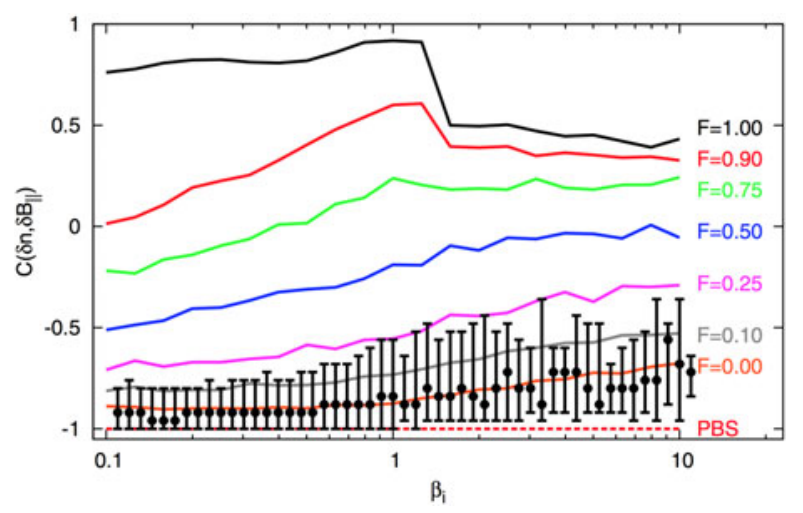

FIgURE 6. Correlation $C$ of density and parallel magnetic fluctuations as a function of $\beta_{i}$, compared to theoretical predictions for a spectrum of kinetic fast and slow mode waves with different fractions $F$ of fast mode to total energy (from Howes et al. 2012).

\subsection{Nature of the compressive fluctuations}

It is also of interest to determine what type of compressive fluctuations are present, i.e. which wave modes they resemble, and whether these require a kinetic description. Klein et al. (2012) showed that the kinetic fast and slow modes retain qualitative characteristics of their MHD counterparts, but differ quantitatively so that a kinetic description is necessary. In particular, $\delta n$ and $\delta B_{\|}$remain predominantly anti-correlated for the slow mode and correlated for the fast mode, with a characteristic $\beta_{i}$ dependence. Howes et al. (2012) compared solar wind measurements of this correlation to that produced from a critically balanced spectrum of kinetic slow waves plus an isotropic spectrum of kinetic fast waves, with different fractions of the two. The results are given in figure 6 and show a strong, $\beta_{i}$-dependent anti-correlation, consistent with the curve in which only slow modes, and not fast modes, contribute to the compressive power. The scarcity of fast mode fluctuations has some interesting implications. Firstly, it helps justify the application of low-frequency approximations, such as gyrokinetics (Schekochihin et al. 2009) and kinetic reduced MHD (Kunz et al. 2015) to the solar wind. Secondly, it suggests that there can be little transfer of energy to whistler turbulence at sub-ion scales (consistent with observations; §4.3), which in turn constrains the possible dissipation mechanisms $(\S 5.1)$.

\subsection{Anisotropy and damping}

An interesting question is why there appears to be a cascade of compressive fluctuations at all, when they are expected to be strongly damped in a $\beta_{i} \sim 1$ plasma (Barnes 1966). In particular, their damping rate is $\gamma \sim k_{\|} v_{A}$, comparable to the time scale on which the Alfvénic turbulence would cascade them to small scales. The solution to this may lie in their anisotropy: if they are significantly more anisotropic than the Alfvénic turbulence, i.e. their $k_{\|}$remains small, they are not strongly damped. So what do we expect the anisotropy of passively mixed compressive fluctuations to be? Lithwick \& Goldreich (2001) suggested that the slow modes inherit the anisotropy of the Alfvénic turbulence that mixes them. However, Schekochihin et al. (2009) argued that the kinetic equation for the compressive fluctuations becomes linear in the Lagrangian frame of the Alfvénic turbulence, and, therefore, 

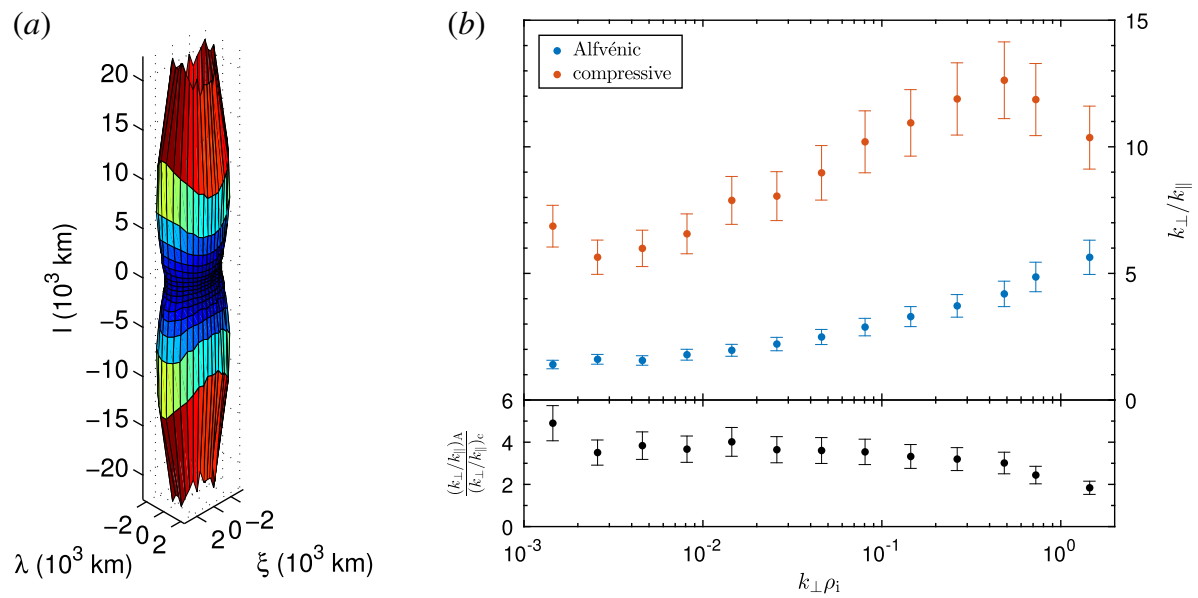

FIGURE 7. (a) 3-D eddy shape for the $\delta|\boldsymbol{B}|$ component of the turbulence at small scales $\left(k_{\perp} \rho_{i} \approx 0.4\right)$ in the same form as figure 3 (from Chen et al. 2012a). (b) Comparison between the anisotropy of the Alfvénic and compressive components of the turbulence.

that they have no parallel cascade. This would lead to highly elongated, compressive structures, with very small $k_{\|}$, and could explain why they are not strongly damped.

The 3-D shape of the $\delta|\boldsymbol{B}|$ fluctuations was measured (using the technique described in $\$ 2.4)$ by Chen et al. (2012a) and is shown in figure 7, where it can be seen that they are indeed very anisotropic. They are, in fact, more anisotropic than the Alfvénic turbulence, as illustrated in figure 7 where the anisotropy $k_{\perp} / k_{\|}$of each component is shown as a function of scale. The ratio of the anisotropy of the compressive to Alfvénic component is shown in the lower panel and can be seen to take a value between 3 and 4 throughout most of the inertial range ${ }^{16}$. Due to the angular resolution of the measurements, this is a lower limit: the compressive fluctuations are at least several times more anisotropic than the Alfvénic turbulence. This is consistent with the prediction of Schekochihin et al. (2009), although there is an alternative interpretation. It is possible that the cascade attempts to generate compressive fluctuations with an anisotropy similar to the Alfvénic turbulence, but these are quickly damped, leaving the highly anisotropic ones to be observed. So the question becomes: are the compressive fluctuations highly anisotropic because the less anisotropic component is damped, or are they generated highly anisotropic to begin with? This remains to be answered, but has some interesting implications, e.g. if damping is taking place, then energy may be being removed from the cascade throughout the inertial range, rather than just at the small scales. However, a recent suggestion by Schekochihin et al. (2016) is that 'anti-phase-mixing' may effectively suppress such damping, providing an alternative explanation for the presence of the compressive fluctuations. Further observations will be required to distinguish these possibilities.

\footnotetext{
${ }^{16}$ At $k_{\perp} \rho_{i} \sim 1$ the compressive component anisotropy reduces, becoming closer to that of the Alfvénic component, consistent with the $\delta B_{\|}$of the Alfvénic turbulence starting to dominate the compressive spectrum.
} 


\section{Kinetic range turbulence}

As the energy cascade proceeds to ever smaller scales, it eventually reaches plasma microscales such as the particle gyroradii and inertial lengths. Important changes occur here. For a start, scale invariance is broken and the energy spectrum deviates from its power-law form, but it is also in this range where dissipation and heating are thought to take place. Several names have been proposed for this range (and the various sub-ranges within), reflecting different possible physical processes. The term 'kinetic range', reflecting the fact that the scales are close to the particle gyroradii, has recently gained popularity and will be used here ${ }^{17}$. Since the resolution of remote astrophysical observations is limited, the solar wind presents an unparalleled opportunity to understand kinetic range turbulence and how it leads to plasma heating. In this section, our recent progress on kinetic range turbulence is discussed.

\subsection{Phenomenological models of kinetic range turbulence}

Similarly to Alfvénic turbulence (\$2.1), phenomenological models have been developed for an energy cascade between ion and electron scales. Early models were based on the fluid equations of electron MHD (EMHD) (e.g. Kingsep, Chukbar \& Yankov 1990), which in fluctuating form are

$$
\frac{\partial \delta \boldsymbol{B}}{\partial t}=\frac{1}{\mu_{0} e n_{e}} \nabla \times\left[\boldsymbol{B}_{0} \times(\nabla \times \delta \boldsymbol{B})+\delta \boldsymbol{B} \times(\nabla \times \delta \boldsymbol{B})\right] .
$$

This is essentially the induction equation with the ions assumed motionless (and electron inertia and dissipation terms also neglected). While this turns out to be a poor assumption for the solar wind kinetic range (see $\S 4.3$ ), the form of the EMHD equations can be used to illustrate some of the basic principles of the kinetic range cascade.

Equation (4.1) is similar to (2.1): there is a linear term (describing whistler waves) and a nonlinear term responsible for the turbulence. The key difference, however, is the additional spatial gradient in both terms. This results in whistler waves being dispersive and leads to a steeper turbulence spectrum. This was first shown by Vaǐnshteǐn (1973), who considered isotropic strong EMHD turbulence. From the form of the nonlinear term in (4.1) it can be seen that the nonlinear time is $\tau_{n l} \propto l^{2} / \delta B$. Assuming a constant energy cascade rate $\varepsilon \propto \delta B^{2} / \tau_{n l} \propto \delta B^{3} / l^{2}$ leads to a magnetic energy spectrum

$$
E_{B}(k) \propto k^{-7 / 3},
$$

steeper than any of the predicted spectra for MHD turbulence. Similarly to MHD turbulence, though, the assumption of isotropy is not robust. When a critical balance between the whistler time scale and nonlinear time scale is assumed, the scale-dependent anisotropy $k_{\|} \propto k_{\perp}^{1 / 3}$ is obtained (Cho \& Lazarian 2004), a stronger anisotropy than for Alfvénic turbulence. This results in anisotropic spectra

$$
E_{B}\left(k_{\perp}\right) \propto k_{\perp}^{-7 / 3}, \quad E_{B}\left(k_{\|}\right) \propto k_{\|}^{-5},
$$

as illustrated in figure 8 .

\footnotetext{
${ }^{17}$ Although this terminology is not ideal, since aspects of the turbulence in the 'kinetic range' can be captured by fluid models, and aspects of turbulence in the larger-scale (MHD) range require a kinetic treatment $(\S 3)$.
} 


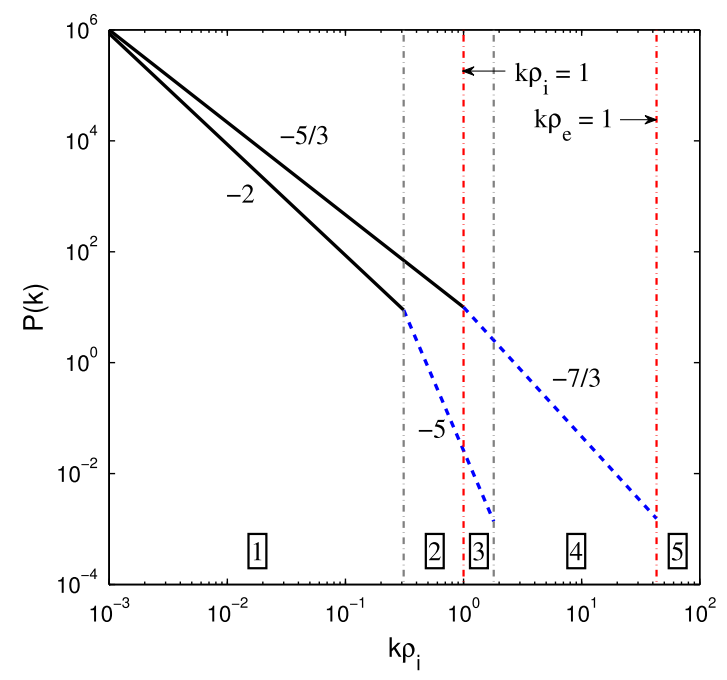

FIGURE 8. Schematic of parallel and perpendicular energy spectra for critically balanced Alfvénic turbulence $\left(k_{\perp}^{-5 / 3}\right.$ and $\left.k_{\|}^{-2}\right)$ at $k_{\perp} \rho_{i}<1$, and kinetic Alfvén or whistler turbulence $\left(k_{\perp}^{-7 / 3}\right.$ and $\left.k_{\|}^{-5}\right)$ at $k_{\perp} \rho_{i}>1$, without intermittency or other corrections (from Chen et al. $2010 b)$.

It was proposed (e.g. Leamon et al. 1998; Howes et al. 2008; Schekochihin et al. 2009; Boldyrev \& Perez 2012), however, that Alfvénic turbulence at large scales transitions to kinetic Alfv́en turbulence at sub-ion scales, rather than whistler/EMHD turbulence, reflecting the fact that the kinetic Alfvén wave (KAW) is the continuation of the oblique Alfvén mode in this regime. The nonlinear fluid equations that capture kinetic Alfvén turbulence between the ion and electron gyroscales $1 / \rho_{i} \ll k_{\perp} \ll 1 / \rho_{e}$ (e.g. Schekochihin et al. 2009; Boldyrev et al. 2013) take the same mathematical structure as the EMHD equations for a strong mean field and anisotropy $k_{\perp} \gg k_{\|}$ (Boldyrev et al. 2013), so the above scaling predictions for the magnetic fluctuations also apply. A key physical difference, however, is that kinetic Alfvén turbulence is low frequency, $\omega \ll k_{\perp} v_{t h, i}$, (whereas whistler turbulence has $\omega \gg k_{\perp} v_{t h, i}$ ) so unlike whistler turbulence, density fluctuations are non-negligible (Chen et al. 2013b; Boldyrev et al. 2013). From the nonlinear kinetic Alfvén equations, the scaling of the magnetic, electric and density fluctuations are

$$
E_{B}\left(k_{\perp}\right) \propto k_{\perp}^{-7 / 3}, \quad E_{E}\left(k_{\perp}\right) \propto k_{\perp}^{-1 / 3}, \quad E_{n}\left(k_{\perp}\right) \propto k_{\perp}^{-7 / 3} .
$$

A modification of these predictions was suggested by Boldyrev \& Perez (2012), who observed strong intermittency in their kinetic Alfvén turbulence simulations, with fluctuation energy concentrated in 2-D sheets. Assuming that the cascade is only active within these sheets (so that the active volume fraction is proportional to the scale of the fluctuations), a weaker anisotropy is obtained, $k_{\|} \propto k_{\perp}^{2 / 3}$ and the total energy spectra are

$$
E\left(k_{\perp}\right) \propto k_{\perp}^{-8 / 3}, \quad E\left(k_{\|}\right) \propto k_{\|}^{-7 / 2} .
$$

As for Alfvénic turbulence, these predictions for the spectra, nature and intermittency of the fluctuations can be directly tested with solar wind observations. 


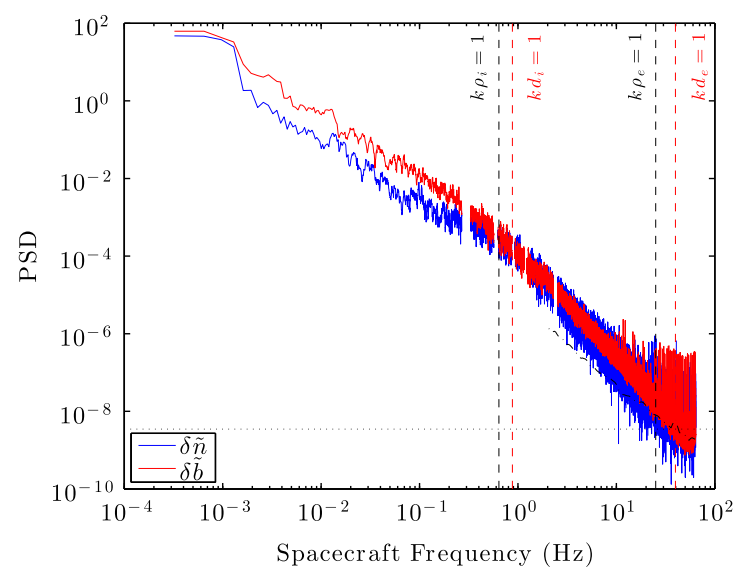

FIGURE 9. Spectra of density and magnetic fluctuations normalised according to (4.6); the vertical dashed lines correspond to the ion and electron gyroradii and inertial length scales under the assumption of the Taylor hypothesis (from Chen et al. 2013a).

\subsection{Kinetic range spectra}

It has been known for a long time that the spectrum of magnetic fluctuations in the solar wind steepens at ion scales (e.g. Coleman 1968; Russell 1972), but it is only recently that we have been able to more comprehensively diagnose this range, using new high-resolution spacecraft instrumentation. While the shape of the magnetic spectrum close to ion scales and close to electron scales is somewhat variable, the range in between is generally found to be a power law with a typical spectral index around -2.8 (e.g. Alexandrova et al. 2009; Sahraoui et al. 2013). More recently, we have also been able to measure the density spectrum in this range (Chen et al. 2012b; Šafránková et al. 2013, 2015). Chen et al. (2012b) showed that this also takes a powerlaw form, with a spectral index of $-2.75 \pm 0.06$, similar to the magnetic spectrum. Typical spectra of the density and magnetic fluctuations are shown in figure 9. Electric field spectra have also been reported to flatten at ion scales (Bale et al. 2005; Sahraoui et al. 2009; Salem et al. 2012) and ion velocity and temperature spectra to steepen (Šafránková et al. 2013, 2016).

The fact that the density and magnetic fluctuations have the same scaling as each other is consistent with expectations for kinetic Alfvén turbulence, in which these two fields are directly coupled (Schekochihin et al. 2009; Boldyrev et al. 2013), but the spectral index of -2.8 is steeper than the pure cascade prediction of $-7 / 3(4.4)^{18}$. Several explanations have been proposed to explain the steeper scaling. For example, Howes et al. (2011) also observed a $k_{\perp}^{-2.8}$ magnetic spectrum in their gyrokinetic simulations, interpreting the steeper-than- $k_{\perp}^{-7 / 3}$ result as due to the presence of electron Landau damping. The steeper spectrum, however, has also been observed in simulations that do not contain such damping (Boldyrev \& Perez 2012; Franci et al. 2015). As mentioned in $\$ 4.1$, Boldyrev \& Perez (2012) suggested that the accumulation of the cascade into intermittent 2-D structures would lead to a $k_{\perp}^{-8 / 3}$ spectrum, which is closer to the observed value. Other explanations have also

\footnotetext{
${ }^{18}$ This comparison assumes the Taylor hypothesis, which is thought to be valid for kinetic Alfvén turbulence at 1 AU but not whistler turbulence (Howes, Klein \& TenBarge 2014; Klein, Howes \& TenBarge 2014).
} 

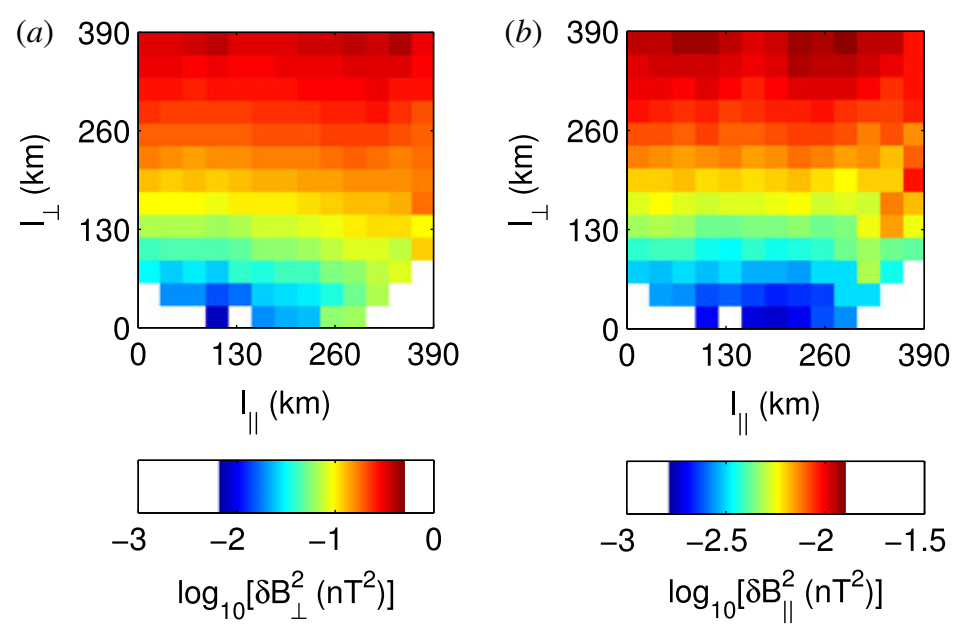

FIGURE 10. Fluctuation power in the perpendicular $(a)$ and parallel $(b)$ magnetic field components as a function of perpendicular $\left(l_{\perp}\right)$ and parallel $\left(l_{\|}\right)$length scale, for the kinetic range between the ion and electron gyroscales, $1 / \rho_{i}<k<1 / \rho_{e}$ (from Chen et al. 2010a).

been proposed (e.g. Meyrand \& Galtier 2013; Passot \& Sulem 2015). It remains to be determined which combination of these, or other possibilities, are responsible for the steep kinetic range spectra.

\subsection{Nature of the fluctuations}

To understand how the kinetic-scale cascade operates and leads to plasma heating, it is necessary to determine the nature of the fluctuations which constitute the turbulence. As mentioned in $\S 2$, turbulence at large scales, $k \rho_{i} \ll 1$, is predominantly Alfvénic, i.e. the fluctuations are polarised similarly to Alfvén waves (Belcher \& Davis 1971). The plasma modes which exist in the kinetic range, however, are more complex. To narrow down the possibilities, it is first helpful to determine the anisotropy of the turbulence, i.e. whether it is dominated by parallel $\left(k_{\|} \gg k_{\perp}\right)$, isotropic $\left(k_{\perp} \sim k_{\|}\right)$or perpendicular $\left(k_{\perp} \gg k_{\|}\right)$fluctuations. Chen et al. (2010a) measured the anisotropy of the magnetic fluctuations in the kinetic range using a technique similar to that described in $\S 2.2$ but with multiple spacecraft to simultaneously measure different directions. The result of this analysis is given in figure 10, which shows the power in the perpendicular and parallel field components as a function of parallel and perpendicular scale. For both components, the power contours are elongated in the parallel direction, indicating that the turbulence consists of perpendicular fluctuations, $k_{\perp} \gg k_{\|}$. This anisotropy is similar to that of the Alfvénic turbulence at large scales, and is consistent with the predictions of $\S 4.1$ that kinetic turbulence remains anisotropic.

The two perpendicular electromagnetic waves that can exist in an isotropic $\beta \sim 1$ plasma for $1 / \rho_{i} \ll k_{\perp} \ll 1 / \rho_{e}$ are the kinetic Alfvén wave and the oblique whistler wave (e.g. TenBarge et al. 2012; Boldyrev et al. 2013) and the models in $\S 4.1$ describe the nonlinear turbulence based on these modes. Chen et al. (2013b) developed a method to distinguish between these two types of turbulence, based on the relative level of density and magnetic fluctuations. The spectra in figure 9 are 
of the normalised fluctuations,

$$
\delta \tilde{n}=\sqrt{\frac{\beta_{i}}{2}\left(1+\frac{T_{e}}{T_{i}}\right)\left[1+\frac{\beta_{i}}{2}\left(1+\frac{T_{e}}{T_{i}}\right)\right]} \frac{\delta n}{n_{0}}, \quad \delta \tilde{\boldsymbol{b}}=\frac{\delta \boldsymbol{B}}{B_{0}} .
$$

Similarly to the Alfvén ratio ( $\S 2.3)$, the kinetic Alfvén ratio can be defined as $r_{K A W}=$ $\delta \tilde{n}^{2} / \delta \tilde{b}_{\perp}^{2}$, which is $r_{K A W}=1$ for a kinetic Alfvén wave (due to its pressure-balanced nature) and $r_{K A W} \ll 1$ for an oblique whistler wave (due to its high frequency $\omega \gg$ $\left.k_{\perp} v_{t h, i}\right)$. It can be seen from figure 9 that in the range between ion and electron scales, the density and magnetic fluctuations are of similar amplitude, and $r_{K A W} \sim$ 1. This suggests that the turbulence is predominantly kinetic Alfvén in nature, with whistler fluctuations making up (at most) a small fraction, which is consistent with the transition from Alfvénic turbulence at larger scales. Chen et al. (2013b) measured the average kinetic Alfvén ratio to be $r_{K A W}=0.75$ in the solar wind and $r_{K A W}=0.79$ in a kinetic Alfvén turbulence simulation, indicating that while the turbulence follows the linear expectation to order unity, the nonlinearities introduce quantitative differences (see also § 2.3).

The kinetic Alfvén nature of the turbulence is consistent with the flattening of the density spectrum seen to occur just before ion scales (e.g. figure 9). The flattening can be explained as the kinetic Alfvén component at small scales taking over from the compressive non-Alfvénic component at larger scales (Harmon \& Coles 2005; Chandran et al. 2009), a model which is consistent with solar wind observations (Chen et al. 2013c). It is also consistent with a significant, rather than negligible, $\delta E_{\|}$spectrum (Mozer \& Chen 2013), although the $\delta E_{\|} / \delta E_{\perp}$ ratio measured by Mozer $\&$ Chen (2013) is larger than the linear prediction ${ }^{19}$. Finally, the measured ion-scale spectral break can be compared to the expected dispersive scale for the transition from Alfvénic to kinetic Alfvén turbulence. Chen et al. (2014a) examined periods of extreme $\beta_{i}$ so that the various ion scales could be distinguished, and found that for $\beta_{i} \gg 1$ the break occurs close to the ion gyroscale $\left(k \rho_{i} \sim 1\right)$, which is indeed the dispersive scale for Alfvénic turbulence, but at $\beta_{i} \ll 1$ it occurs at the ion inertial length $\left(k d_{i} \sim 1\right)$. This latter result remains to be explained, but one possibility is the presence of a significant $k_{\|}$component in low- $\beta_{i}$ turbulence (Boldyrev et al. 2015).

\subsection{Intermittency}

An important feature of turbulence, alluded to in $\S 4.1$, is intermittency, i.e. the nonGaussian nature of the fluctuations. Intermittency has been well studied in the solar wind for $k \rho_{i}<1$ (see reviews by Alexandrova et al. 2013; Bruno \& Carbone 2013), where most quantities are seen to become increasingly non-Gaussian towards smaller scales, a well-known feature of turbulence. This is related to the turbulent energy being concentrated into a smaller fraction of the volume as the cascade proceeds to smaller scales, and results in the formation of energetic structures within the plasma. Understanding this intermittency and structure generation is important for both the cascade process and plasma heating, since the energy dissipation is thought to be focussed at these structures.

As a result of the Alfvénic cascade, the probability density functions (PDFs) of the fluctuations are significantly non-Gaussian by the time ion scales $k \rho_{i} \sim 1$

\footnotetext{
${ }^{19}$ This remains to be explained but may be either a nonlinear effect or related to the fact that the periods studied were unusually low amplitude, in order for $\delta E_{\|}$to be obtained from a two-component electric field measurement.
} 

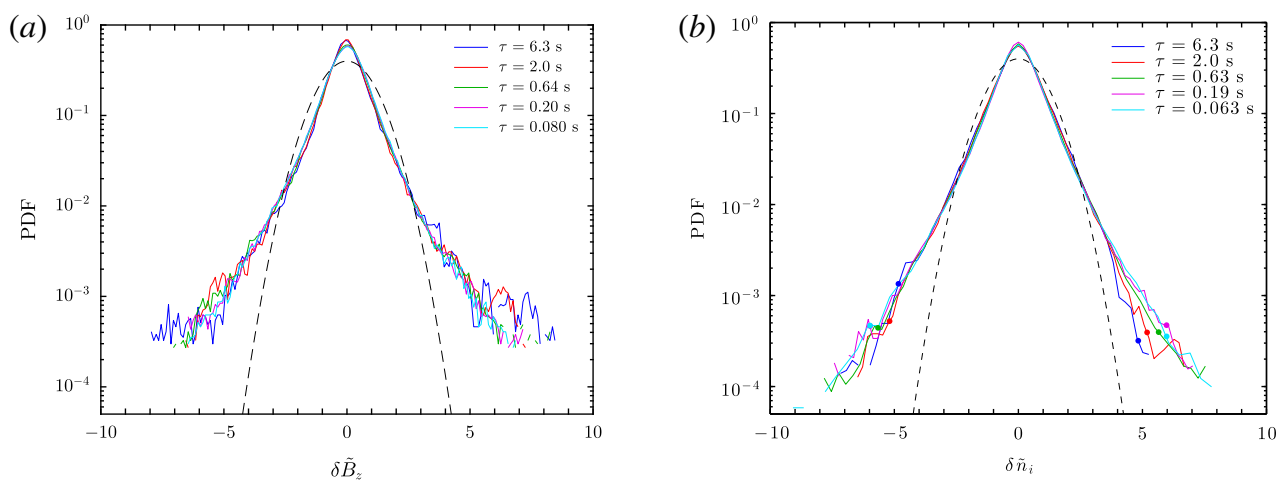

FIgURE 11. (a) PDFs of magnetic fluctuations from ion to electron scales (using the data interval of Chen et al. 2010a). (b) Same for density fluctuations (from Chen et al. 2014b). A Gaussian distribution is given by the black dashed line.

are reached. Recently, it has been possible to investigate how the PDF shape continues to change down to electron scales. Intriguingly, Kiyani et al. (2009) showed that from ion to electron scales, $1 / \rho_{i}<k<1 / \rho_{e}$, the rescaled PDFs of magnetic fluctuations do not get significantly more non-Gaussian, but retain a similar shape. Chen et al. (2014b) found a similar behaviour for the density fluctuation PDFs, which are shown in figure 11, along with the magnetic fluctuations ${ }^{20}$. The similar behaviour of the density and magnetic fluctuations in the kinetic range is expected, since in kinetic Alfvén turbulence these fields are directly coupled $(\$ 4.1)$, although the self-similarity of the PDFs is unusual for a turbulent cascade. One possibility is that kinetic range turbulence is inherently mono-fractal; although a recent measurement of the multi-fractal spectrum by Sorriso-Valvo et al. (2016) showed a larger multi-fractality in the the kinetic range. Another possibility is that the kurtosis of the fluctuations is limited by another process, such as the instability of the current sheet structures which may form (Biskamp, Welter \& Walter 1990). However, the nature of the intermittency in the kinetic range remains to be fully understood.

An alternative way to examine intermittency is through the distribution of rotation angles of the magnetic field. The rotation angle $\alpha$ is defined as the angle through which the field rotates over a given spatial scale (or time scale $\tau$ measured by a spacecraft under the Taylor hypothesis),

$$
\alpha(t, \tau)=\cos ^{-1}\left[\frac{\boldsymbol{B}(t) \cdot \boldsymbol{B}(t+\tau)}{|\boldsymbol{B}(t)||\boldsymbol{B}(t+\tau)|}\right],
$$

and has been used to study both the intermittency and the structures that are formed (e.g. Zhdankin, Boldyrev \& Mason 2012, and references therein). Such analysis was recently extended into the kinetic range by Chen et al. (2015), in which the distribution of $\alpha$ over scales $1 / \rho_{i}<k<1 / \rho_{e}$ was investigated. It was found that, similar to larger scales (Bruno et al. 2004; Zhdankin et al. 2012), the PDFs of $\alpha$ are well fit by log-normal distributions, with only small changes in shape from ion to electron scales.

\footnotetext{
${ }^{20}$ Note that the density and magnetic fluctuations in this figure are from different time periods, which can account for the different absolute levels of non-Gaussianity.
} 

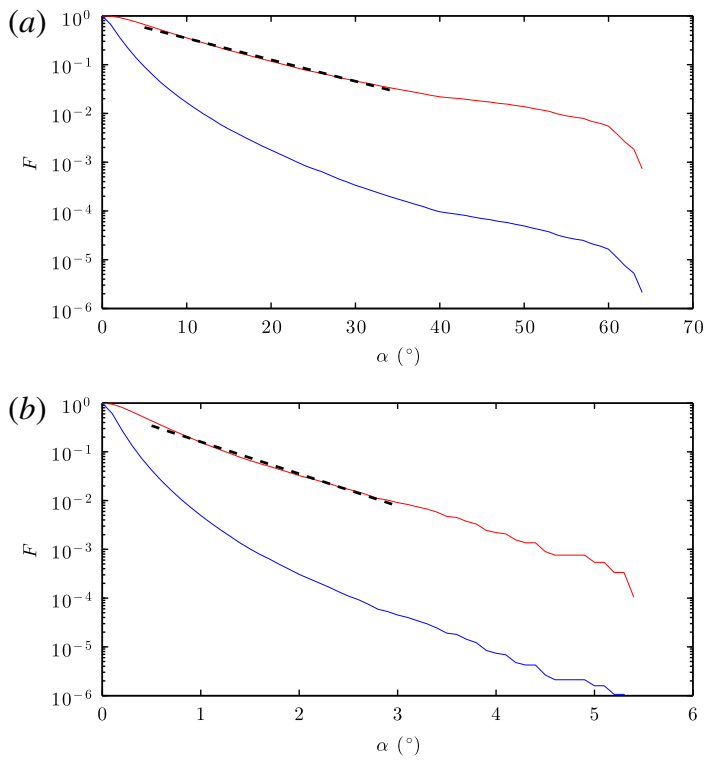

FIGURE 12. Fraction of magnetic rotations larger than $\alpha$ (blue) and magnetic fluctuation energy in those angles (red) at $(a)$ ion and $(b)$ electron scales, along with exponential fits (black dashed) (from Chen et al. 2015).

The large rotations are sometimes identified as the 'structures', so it is of interest to investigate their distribution, how much energy they contain and how large the rotations are. Figure 12 shows the fraction of the time that the rotation angles are larger than $\alpha$, as a function of $\alpha$, for scales $\tau$ corresponding to the ion and electron gyroscales. Also shown is the fraction of magnetic fluctuation energy $|\delta \boldsymbol{B}|^{2}$ contained in these angles. It can be seen that for a given angle, the energy fraction is much larger than the filling fraction, consistent with the non-Gaussian nature of the fluctuations. Similar analyses have been performed for the energy dissipation at current structures in both MHD and kinetic simulations (Wan et al. 2012, 2016; Zhdankin et al. 2014; Zhdankin, Boldyrev \& Uzdensky 2016b), with similar results. However, at kinetic scales, the absolute rotation angles are relatively small. The energy fraction in figure 12 falls exponentially, with $e$-folding angle $9.8^{\circ}$ at ion scales and $0.66^{\circ}$ at electron scales, demonstrating that large angles $>30^{\circ}$ do not contain significant energy in the kinetic range. This places important constraints on energy conversion and dissipation mechanisms, such as magnetic reconnection, that have been proposed to be taking place here.

\section{Dissipation and future prospects}

While we have made significant progress in recent years, there are still many aspects of astrophysical plasma turbulence which remain to be understood. As well as the questions mentioned in the above sections, one of the major unsolved problems is how the turbulent energy is finally transferred to the particles and dissipated, i.e. how astrophysical plasmas, which are usually considered highly collisionless on the spatial and temporal scales of the turbulence, can be heated to the temperatures we observe. Work has begun on this question, but it will likely be from future measurements and new space missions that this problem is finally solved. In this section, a selection of that recent work, and possible future directions, are discussed. 


\subsection{Dissipation}

One basic question is how dissipation is distributed throughout a turbulent plasma, i.e. to what extent it is focussed at the energetic structures generated by intermittency. In fact, measuring the distribution of the dissipation can, in some sense, be considered a more direct way of measuring the intermittency of the cascade, since it is more directly connected to the energy cascade rate ${ }^{21}$. Zhdankin, Boldyrev \& Chen (2016a) measured the PDFs of the dissipation, averaged over different MHD range scales, in both MHD simulations and the solar wind (where a proxy based on magnetic field measurements was used). The PDFs were found to be well fitted by log-normal distributions in all cases $^{22}$, although the higher-order moments of both the solar wind and simulated distributions were better described by log-Poisson, rather than log-normal, scaling predictions. The rate at which these PDFs broaden towards smaller scales indicates the level of intermittency, and this was found to be consistent between the solar wind and simulations and indicative of significant intermittency of the energy dissipation. It is of interest to determine which types of structures this dissipation is focussed at, and whether these correspond to any well-known plasma phenomena. For example, reconnecting current sheets (Retinò et al. 2007; Sundkvist et al. 2007), Alfvén vortices (Alexandrova et al. 2006), double layers (Stawarz, Ergun \& Goodrich 2015) and several others have been proposed. It remains to be determined which of these, if any, play a significant role in the dissipation of plasma turbulence.

A more fundamental, and even less well understood, question is the nature of the physical mechanisms which transfer energy from the electromagnetic turbulence to the particles and lead to the irreversible heating of the plasma. Several possibilities have been proposed, including ion cyclotron damping (e.g. Coleman 1968; Smith, Vasquez \& Hollweg 2012), Landau damping (e.g. Howes et al. 2008; TenBarge \& Howes 2013), stochastic heating (e.g. Chandran et al. 2010), entropy cascade (Schekochihin et al. 2009) and reconnection associated mechanisms (e.g. Egedal, Daughton \& Le 2012; Drake \& Swisdak 2014). Identifying which combinations of these operate, under which conditions, will likely involve detailed examination of particle distributions. While future missions may be required to fully answer this (§ 5.2), there have been some initial hints in the existing data. For example, He et al. (2015b) interpreted the contours of measured ion distributions as being consistent with quasilinear expectations for the cyclotron and Landau resonances, and $\mathrm{He}$ et al. (2015a) found plateaus in the distributions at the phase speeds of the Alfvén and slow mode waves, suggesting possible resonant damping of these modes. Various other indirect evidence has also been reported for some of the other mechanisms, although it will likely be from more direct techniques (e.g. Klein \& Howes 2016) that the answer to this question is finally determined.

\subsection{Future missions}

In the coming years, we are set to continue understanding more about turbulence and heating in space and astrophysical plasmas, through several new missions which are due to be launched soon, or have been proposed. The Magnetospheric Multiscale (MMS) mission (Burch et al. 2016), launched in 2015, is already producing important new results, in particular from the increased time resolution of the ion and electron

\footnotetext{
${ }^{21}$ Relating the fluctuations of the energy dissipation to those of the turbulent fields requires an additional assumption, i.e. the plasma turbulence equivalent of the Kolmogorov (1962) refined similarity hypothesis (see, e.g. Chandran et al. 2015).

${ }^{22} \mathrm{~A} \log$-normal was also found to fit the distribution of the local energy transfer rate in the solar wind (Sorriso-Valvo et al. 2015).
} 
measurements (Pollock et al. 2016), in the Earth's magnetosphere and magnetosheath. In 2017, the apogee of the MMS orbit is due to be raised (Fuselier et al. 2016), so that for the extended mission, it will spend significant time in the free solar wind, allowing the study of kinetic turbulence there in even greater detail.

The following year (2018) is due to see the launch of Solar Probe Plus (Fox et al. 2015) and Solar Orbiter (Müller et al. 2013), which will both travel closer to the Sun than any previous spacecraft, and for which turbulence, along with the associated heating, is one of the main science goals. In particular, Solar Probe Plus will travel to within 9 Solar radii of the solar surface, and will have a comprehensive payload measuring the electromagnetic fields (Bale et al. 2016) and particles (Kasper et al. 2015) to explore turbulence and heating within the corona for the first time. At the same time, the Voyager spacecraft are moving further away from the Sun, allowing the first in situ measurements of turbulence in the local interstellar medium (Burlaga, Florinski \& Ness 2015).

Finally, Turbulence Heating ObserverR (THOR) (Vaivads et al. 2016) is a mission concept proposed to the European Space Agency that is currently in study phase, and if selected would be due to launch in 2026. The mission will have the highestresolution set of instruments to date and will explore the range of near-Earth space environments to understand how turbulent energy is dissipated, how that energy is partitioned and how this operates in different turbulence regimes. THOR would be the first space mission dedicated to the topic of turbulent plasma heating, with broad implications for our understanding of astrophysical plasmas. Therefore, the coming years look very promising for the study of turbulence and heating in a variety of astrophysical environments.

\section{Acknowledgements}

I would like to thank my collaborators who have worked with me on the projects discussed in this paper. I was supported by an STFC Ernest Rutherford Fellowship and an Imperial College Junior Research Fellowship.

\section{REFERENCES}

Alexandrova, O., Chen, C. H. K., Sorriso-Valvo, L., Horbury, T. S. \& Bale, S. D. 2013 Solar wind turbulence and the role of ion instabilities. Space Sci. Rev. 178, 101.

Alexandrova, O., Mangeney, A., Maksimovic, M., Cornilleau-Wehrlin, N., Bosqued, J.-M. \& ANDRÉ, M. 2006 Alfvén vortex filaments observed in magnetosheath downstream of a quasi-perpendicular bow shock. J. Geophys. Res. 111, 12208.

Alexandrova, O., Saur, J., Lacombe, C., Mangeney, A., Mitchell, J., Schwartz, S. J. \& ROBERT, P. 2009 Universality of solar-wind turbulent spectrum from MHD to electron scales. Phys. Rev. Lett. 103, 165003.

Armstrong, J. W., Rickett, B. J. \& Spangler, S. R. 1995 Electron density power spectrum in the local interstellar medium. Astrophys. J. 443, 209.

BAlbus, S. A. \& HAWLEY, J. F. 1998 Instability, turbulence, and enhanced transport in accretion disks. Rev. Mod. Phys. 70, 1.

Bale, S. D., Kellogg, P. J., Mozer, F. S., Horbury, T. S. \& Reme, H. 2005 Measurement of the electric fluctuation spectrum of magnetohydrodynamic turbulence. Phys. Rev. Lett. 94, 215002 .

Bale, S. D., Goetz, K., Harvey, P. R., Turin, P., Bonnell, J. W., Dudok De Wit, T., Ergun, R. E., MacDowall, R. J., Pulupa, M., Andre, M. et al. 2016 The FIELDS instrument suite for solar probe plus - measuring the coronal plasma and magnetic field, plasma waves and turbulence, and radio signatures of solar transients. Space Sci. Rev. doi:10.1007/s11214-016-0244-5. 
Barnes, A. 1966 Collisionless damping of hydromagnetic waves. Phys. Fluids 9, 1483.

Belcher, J. W. \& DAVIS, L. JR. 1971 Large-amplitude Alfvén waves in the interplanetary medium, 2. J. Geophys. Res. 76, 3534.

BERESNYAK, A. 2015 On the parallel spectrum in magnetohydrodynamic turbulence. Astrophys. J. 801, L9.

Beresnyak, A. \& Lazarian, A. 2008 Strong imbalanced turbulence. Astrophys. J. 682, 1070.

Biskamp, D., Welter, H. \& WAlter, M. 1990 Statistical properties of two-dimensional magnetohydrodynamic turbulence. Phys. Fluids B 2, 3024.

Boldyrev, S. 2006 Spectrum of magnetohydrodynamic turbulence. Phys. Rev. Lett. 96, 115002.

Boldyrev, S., Chen, C. H. K., XiA, Q. \& Zhdankin, V. 2015 Spectral breaks of Alfvénic turbulence in a collisionless plasma. Astrophys. J. 806, 238.

Boldyrev, S., Horaites, K., XiA, Q. \& Perez, J. C. 2013 Toward a theory of astrophysical plasma turbulence at subproton scales. Astrophys. J. 777, 41.

Boldyrev, S. \& Perez, J. C. 2012 Spectrum of kinetic-Alfvén turbulence. Astrophys. J. 758, L44.

Boldyrev, S., Perez, J. C. \& WANG, Y. $2012 a$ Residual energy in weak and strong MHD turbulence. ASP Conf. Ser. 459, 3.

Boldyrev, S., Perez, J. C. \& Zhdankin, V. $2012 b$ Residual energy in MHD turbulence and in the solar wind. AIP Conf. Proc. 1436, 18.

Bruno, R. \& Carbone, V. 2013 The solar wind as a turbulence laboratory. Living Rev. Sol. Phys. $10,2$.

Bruno, R., Carbone, V., Primavera, L., Malara, F., Sorriso-Valvo, L., Bavassano, B. \& VELTRI, P. 2004 On the probability distribution function of small-scale interplanetary magnetic field fluctuations. Ann. Geophys. 22, 3751.

Burch, J. L., Moore, T. E., Torbert, R. B. \& Giles, B. L. 2016 Magnetospheric multiscale overview and science objectives. Space Sci. Rev. 199, 5.

Burlaga, L. F., Florinski, V. \& Ness, N. F. 2015 In situ observations of magnetic turbulence in the local interstellar medium. Astrophys. J. 804, L31.

Chandran, B. D. G. 2008 Strong anisotropic MHD turbulence with cross helicity. Astrophys. J. $685,646$.

Chandran, B. D. G., Li, B., Rogers, B. N., Quataert, E. \& Germaschewski, K. 2010 Perpendicular ion heating by low-frequency Alfvén-wave turbulence in the solar wind. Astrophys. J. 720, 503.

Chandran, B. D. G., Quataert, E., Howes, G. G., Xia, Q. \& Pongkitiwanichakul, P. 2009 Constraining low-frequency Alfvénic turbulence in the solar wind using density-fluctuation measurements. Astrophys. J. 707, 1668.

Chandran, B. D. G., Schekochinin, A. A. \& Mallet, A. 2015 Intermittency and alignment in strong RMHD turbulence. Astrophys. J. 807, 39.

Chen, C. H. K., Bale, S. D., Salem, C. \& Mozer, F. S. $2011 a$ Frame dependence of the electric field spectrum of solar wind turbulence. Astrophys. J. 737, L41.

Chen, C. H. K., Bale, S. D., Salem, C. S. \& Maruca, B. A. $2013 a$ Residual energy spectrum of solar wind turbulence. Astrophys. J. 770, 125.

Chen, C. H. K., Boldyrev, S., XiA, Q. \& Perez, J. C. $2013 b$ Nature of subproton scale turbulence in the solar wind. Phys. Rev. Lett. 110, 225002.

Chen, C. H. K., Horbury, T. S., Schekochinin, A. A., Wicks, R. T., Alexandrova, O. \& Mitchell, J. 2010a Anisotropy of solar wind turbulence between ion and electron scales. Phys. Rev. Lett. 104, 255002.

Chen, C. H. K., Howes, G. G., Bonnell, J. W., Mozer, F. S., Klein, K. G. \& Bale, S. D. 2013c Kinetic scale density fluctuations in the solar wind. AIP Conf. Proc. 1539, 143.

Chen, C. H. K., Leung, L., Boldyrev, S., Maruca, B. A. \& Bale, S. D. $2014 a$ Ion-scale spectral break of solar wind turbulence at high and low beta. Geophys. Res. Lett. 41, 8081.

Chen, C. H. K., Mallet, A., Schekochihin, A. A., Horbury, T. S., Wicks, R. T. \& Bale, S. D. $2012 a$ Three-dimensional structure of solar wind turbulence. Astrophys. J. 758, 120. 
Chen, C. H. K., Mallet, A., Yousef, T. A., Schekochinin, A. A. \& Horbury, T. S. $2011 b$ Anisotropy of Alfvénic turbulence in the solar wind and numerical simulations. Mon. Not. R. Astron. Soc. 415, 3219.

Chen, C. H. K., Matteini, L., Burgess, D. \& Horbury, T. S. 2015 Magnetic field rotations in the solar wind at kinetic scales. Mon. Not. R. Astron. Soc. 453, L64.

Chen, C. H. K., Matteini, L., Schekochihin, A. A., Stevens, M. L., Salem, C. S., Maruca, B. A., KunZ, M. W. \& BALE, S. D. 2016 Multi-species measurements of the firehose and mirror instability thresholds in the solar wind. Astrophys. J. 825, L26.

Chen, C. H. K., Salem, C. S., Bonnell, J. W., Mozer, F. S. \& Bale, S. D. $2012 b$ Density fluctuation spectrum of solar wind turbulence between ion and electron scales. Phys. Rev. Lett. 109, 035001.

Chen, C. H. K., Sorriso-VAlvo, L., ŠAfrÁnKovÁ, J. \& NĚMeČEK, Z. 2014b Intermittency of solar wind density fluctuations from ion to electron scales. Astrophys. J. 789, L8.

Chen, C. H. K., Wicks, R. T., Horbury, T. S. \& Schekochinin, A. A. $2010 b$ Interpreting power anisotropy measurements in plasma turbulence. Astrophys. J. 711, L79.

CHO, J. \& LAZARIAN, A. 2004 The anisotropy of electron magnetohydrodynamic turbulence. Astrophys. J. 615, L41.

Cho, J. \& Vishniac, E. T. 2000 The anisotropy of magnetohydrodynamic Alfvénic turbulence. Astrophys. J. 539, 273.

Coleman, P. J. 1968 Turbulence, viscosity, and dissipation in the solar-wind plasma. Astrophys. J. 153, 371.

Cranmer, S. R., Asgari-Targhi, M., Miralles, M. P., Raymond, J. C., Strachan, L., Tian, H. \& Woolsey, L. N. 2015 The role of turbulence in coronal heating and solar wind expansion. Phil. Trans. R. Soc. Lond. A 373, 20140148.

Dasso, S., Milano, L. J., Matthaeus, W. H. \& Smith, C. W. 2005 Anisotropy in fast and slow solar wind fluctuations. Astrophys. J. 635, L181.

DRAKE, J. F. \& SWISDAK, M. 2014 The onset of ion heating during magnetic reconnection with a strong guide field. Phys. Plasmas 21, 072903.

Egedal, J., Daughton, W. \& Le, A. 2012 Large-scale electron acceleration by parallel electric fields during magnetic reconnection. Nat. Phys. 8, 321.

Elsasser, W. M. 1950 The hydromagnetic equations. Phys. Rev. 79, 183.

Fox, N. J., Velli, M. C., Bale, S. D., Decker, R., Driesman, A., Howard, R. A., Kasper, J. C., Kinnison, J., Kusterer, M., LARio, D. et al. 2015 The solar probe plus mission: humanity's first visit to our star. Space Sci. Rev. doi:10.1007/s11214-015-0211-6.

Franci, L., LAndi, S., Matteini, L., Verdini, A. \& Hellinger, P. 2015 High-resolution hybrid simulations of kinetic plasma turbulence at proton scales. Astrophys. J. 812, 21.

Frisch, U., Pouquet, A., Leorat, J. \& Mazure, A. 1975 Possibility of an inverse cascade of magnetic helicity in magnetohydrodynamic turbulence. J. Fluid Mech. 68, 769.

Fuselier, S. A., Lewis, W. S., Schiff, C., Ergun, R., Burch, J. L., Petrinec, S. M. \& Trattner, K. J. 2016 Magnetospheric multiscale science mission profile and operations. Space Sci. Rev. 199, 77.

Galtier, S., Nazarenko, S. V., Newell, A. C. \& Pouquet, A. 2000 A weak turbulence theory for incompressible magnetohydrodynamics. J. Plasma Phys. 63, 447.

Goldreich, P. \& SRIDhar, S. 1995 Toward a theory of interstellar turbulence. II. Strong Alfvénic turbulence. Astrophys. J. 438, 763.

Goldreich, P. \& SRidhar, S. 1997 Magnetohydrodynamic turbulence revisited. Astrophys. J. 485, 680.

Grappin, R., Leorat, J. \& Pouquet, A. 1983 Dependence of MHD turbulence spectra on the velocity field-magnetic field correlation. Astron. Astrophys. 126, 51.

HARmon, J. K. \& COLES, W. A. 2005 Modeling radio scattering and scintillation observations of the inner solar wind using oblique Alfvén/ion cyclotron waves. J. Geophys. Res. 110, 3101. 
He, J., Tu, C., Marsch, E., Chen, C. H. K., Wang, L., Pei, Z., Zhang, L., Salem, C. S. \& BALE, S. D. $2015 a$ Proton heating in solar wind compressible turbulence with collisions between counter-propagating waves. Astrophys. J. 813, L30.

He, J., WAng, L., Tu, C., Marsch, E. \& Zong, Q. $2015 b$ Evidence of Landau and cyclotron resonance between protons and kinetic waves in solar wind turbulence. Astrophys. J. 800, L31.

Higdon, J. C. 1984 Density fluctuations in the interstellar medium: evidence for anisotropic magnetogasdynamic turbulence. I. Model and astrophysical sites. Astrophys. J. 285, 109.

Horbury, T. S., Forman, M. \& OUghton, S. 2008 Anisotropic scaling of magnetohydrodynamic turbulence. Phys. Rev. Lett. 101, 175005.

Horbury, T. S., Wicks, R. T. \& Chen, C. H. K. 2012 Anisotropy in space plasma turbulence: solar wind observations. Space Sci. Rev. 172, 325.

Howes, G. G., Bale, S. D., Klein, K. G., Chen, C. H. K., Salem, C. S. \& Tenbarge, J. M. 2012 The slow-mode nature of compressible wave power in solar wind turbulence. Astrophys. J. 753, L19.

Howes, G. G., Cowley, S. C., Dorland, W., Hammett, G. W., Quataert, E. \& Schekochinin, A. A. 2008 A model of turbulence in magnetized plasmas: implications for the dissipation range in the solar wind. J. Geophys. Res. 113, 5103.

Howes, G. G., Klein, K. G. \& TenBarge, J. M. 2014 Validity of the Taylor hypothesis for linear kinetic waves in the weakly collisional solar wind. Astrophys. J. 789, 106.

Howes, G. G., TenBarge, J. M., Dorland, W., Quataert, E., Schekochinin, A. A., Numata, R. \& TATSUNO, T. 2011 Gyrokinetic simulations of solar wind turbulence from ion to electron scales. Phys. Rev. Lett. 107, 035004.

Iroshnikov, P. S. 1963 Turbulence of a conducting fluid in a strong magnetic field. Astron. Zh. 40, 742 .

Kasper, J. C., Abiad, R., Austin, G., Balat-Pichelin, M., Bale, S. D., Belcher, J. W., Berg, P., Bergner, H., Berthomier, M., Bookbinder, J. et al. 2015 Solar wind electrons alphas and protons (SWEAP) investigation: design of the solar wind and coronal plasma instrument suite for solar probe plus. Space Sci. Rev. doi:10.1007/s11214-015-0206-3.

Kingsep, A. S., Chukbar, K. V. \& YAnkov, V. V. 1990 Electron magnetohydrodynamics. Rev. Plasma Phys. 16, 243.

Kiyani, K. H., Chapman, S. C., Khotyaintsev, Y. V., Dunlop, M. W. \& Sahraoui, F. 2009 Global scale-invariant dissipation in collisionless plasma turbulence. Phys. Rev. Lett. 103, 075006.

KiYAni, K. H., Osman, K. T. \& Chapman, S. C. 2015 Dissipation and heating in solar wind turbulence: from the macro to the micro and back again. Phil. Trans. R. Soc. A 373, 20140155.

Klein, K. G. \& Howes, G. G. 2016 Measuring collisionless damping in heliospheric plasmas using field-particle correlations. Astrophys. J. 826, L30.

Klein, K. G., Howes, G. G. \& TenBarge, J. M. 2014 The violation of the Taylor hypothesis in measurements of solar wind turbulence. Astrophys. J. 790, L20.

Klein, K. G., Howes, G. G., Tenbarge, J. M., Bale, S. D., Chen, C. H. K. \& Salem, C. S. 2012 Using synthetic spacecraft data to interpret compressible fluctuations in solar wind turbulence. Astrophys. J. 755, 159.

Kolmogorov, A. N. 1962 A refinement of previous hypotheses concerning the local structure of turbulence in a viscous incompressible fluid at high Reynolds number. J. Fluid Mech. 13, 82.

Kraichnan, R. H. 1965 Inertial-range spectrum of hydromagnetic turbulence. Phys. Fluids 8, 1385.

Kulsrud, R. M. \& Zweibel, E. G. 2008 On the origin of cosmic magnetic fields. Rep. Prog. Phys. 71, 046901.

Kunz, M. W., Schekochinin, A. A., Chen, C. H. K., Abel, I. G. \& Cowley, S. C. 2015 Inertial-range kinetic turbulence in pressure-anisotropic astrophysical plasmas. J. Plasma Phys. 81, 325810501. 
Leamon, R. J., Smith, C. W., Ness, N. F., Matthaeus, W. H. \& Wong, H. K. 1998 Observational constraints on the dynamics of the interplanetary magnetic field dissipation range. J. Geophys. Res. 103, 4775.

Lithwick, Y. \& GoldReich, P. 2001 Compressible magnetohydrodynamic turbulence in interstellar plasmas. Astrophys. J. 562, 279.

Lithwick, Y., Goldreich, P. \& SRIDhar, S. 2007 Imbalanced strong MHD turbulence. Astrophys. J. 655, 269.

LUO, Q. Y. \& WU, D. J. 2010 Observations of anisotropic scaling of solar wind turbulence. Astrophys. J. 714, L138.

Mallet, A. \& Schekochinin, A. A. 2016 A statistical model of three-dimensional anisotropy and intermittency in strong alfvénic turbulence. arXiv:1606.00466.

Mallet, A., Schekochinin, A. A., Chandran, B. D. G., Chen, C. H. K., Horbury, T. S., WiCKS, R. T. \& GREEnAN, C. C. 2016 Measures of three-dimensional anisotropy and intermittency in strong Alfvénic turbulence. Mon. Not. R. Astron. Soc. 459, 2130.

Mangeney, A., Salem, C., Veltri, P. L. \& Cecconi, B. 2001 Intermittency in the solar wind turbulence and the haar wavelet transform. In ESA Special Pub. 492, Sheffield Space Plasma Meeting: Multipoint Measurements versus Theory (ed. B. Warmbein), p. 53. ESA.

MARON, J. \& GoldREICH, P. 2001 Simulations of incompressible magnetohydrodynamic turbulence. Astrophys. J. 554, 1175.

Matthaeus, W. H. \& Goldstein, M. L. 1986 Low-frequency $1 / f$ noise in the interplanetary magnetic field. Phys. Rev. Lett. 57, 495.

Matthaeus, W. H., Servidio, S., Dmitruk, P., Carbone, V., Oughton, S., Wan, M. \& OSMAN, K. T. 2012 Local anisotropy, higher order statistics, and turbulence spectra. Astrophys. J. 750, 103.

McKee, C. F. \& Ostriker, E. C. 2007 Theory of star formation. Annu. Rev. Astron. Astrophys. 45, 565 .

Meyrand, R. \& Galtier, S. 2013 Anomalous $k_{\perp}^{-8 / 3}$ spectrum in electron magnetohydrodynamic turbulence. Phys. Rev. Lett. 111, 264501.

Montgomery, D. \& TuRner, L. 1981 Anisotropic magnetohydrodynamic turbulence in a strong external magnetic field. Phys. Fluids 24, 825.

Mozer, F. S. \& Chen, C. H. K. 2013 Parallel electric field spectrum of solar wind turbulence. Astrophys. J. 768, L10.

MÜlleR, W.-C. \& GRAPPIN, R. 2005 Spectral energy dynamics in magnetohydrodynamic turbulence. Phys. Rev. Lett. 95, 114502.

Müller, D., Marsden, R. G., St. Cyr, O. C. \& Gilbert, H. R. 2013 Solar orbiter exploring the sun-heliosphere connection. Solar Phys. 285, 25.

Oughton, S., Matthaeus, W. H., Wan, M. \& Osman, K. T. 2015 Anisotropy in solar wind plasma turbulence. Phil. Trans. R. Soc. Lond. A 373, 20140152.

PAssot, T. \& Sulem, P. L. 2015 A model for the non-universal power law of the solar wind sub-ion-scale magnetic spectrum. Astrophys. J. 812, L37.

Perez, J. C. \& Boldyrev, S. 2009 Role of cross-helicity in magnetohydrodynamic turbulence. Phys. Rev. Lett. 102, 025003.

Perez, J. C. \& Chandran, B. D. G. 2013 Direct numerical simulations of reflection-driven, reduced magnetohydrodynamic turbulence from the sun to the Alfvén critical point. Astrophys. J. 776, 124.

Podesta, J. J. \& BhattacharJee, A. 2010 Theory of incompressible magnetohydrodynamic turbulence with scale-dependent alignment and cross-helicity. Astrophys. J. 718, 1151.

Podesta, J. J. \& Borovsky, J. E. 2010 Scale invariance of normalized cross-helicity throughout the inertial range of solar wind turbulence. Phys. Plasmas 17, 112905.

Podesta, J. J., Chandran, B. D. G., Bhattacharjee, A., Roberts, D. A. \& Goldstein, M. L. 2009 Scale-dependent angle of alignment between velocity and magnetic field fluctuations in solar wind turbulence. J. Geophys. Res. 114, A01107.

Podesta, J. J., Roberts, D. A. \& Goldstein, M. L. 2007 Spectral exponents of kinetic and magnetic energy spectra in solar wind turbulence. Astrophys. J. 664, 543. 
Pollock, C., Moore, T., Jacques, A., Burch, J., Gliese, U., Saito, Y., Omoto, T., Avanov, L., BARrie, A., COFFEY, V. et al. 2016 Fast plasma investigation for magnetospheric multiscale. Space Sci. Rev. 199, 331.

Pouquet, A., Frisch, U. \& Leorat, J. 1976 Strong MHD helical turbulence and the nonlinear dynamo effect. J. Fluid Mech. 77, 321.

Retinò, A., Sundkvist, D., Vaivads, A., Mozer, F., André, M. \& Owen, C. J. 2007 In situ evidence of magnetic reconnection in turbulent plasma. Nat. Phys. 3, 236.

Russell, C. T. 1972 Comments on the measurement of power spectra of the interplanetary magnetic field. In NASA Special Pub. 308, Solar Wind (ed. C. P. Sonett, P. J. Coleman \& J. M. Wilcox), p. 365. NASA.

Sahraoui, F., Goldstein, M. L., Robert, P. \& Khotyaintsev, Y. V. 2009 Evidence of a cascade and dissipation of solar-wind turbulence at the electron gyroscale. Phys. Rev. Lett. 102, 231102.

Sahraoui, F., Huang, S. Y., Belmont, G., Goldstein, M. L., Rétino, A., Robert, P. \& DE PAtoul, J. 2013 Scaling of the electron dissipation range of solar wind turbulence. Astrophys. J. 777, 15.

Salem, C. S., Howes, G. G., Sundkvist, D., Bale, S. D., Chaston, C. C., Chen, C. H. K. \& Mozer, F. S. 2012 Identification of kinetic Alfvén wave turbulence in the solar wind. Astrophys. J. 745, L9.

Scalo, J. \& Elmegreen, B. G. 2004 Interstellar turbulence II: implications and effects. Annu. Rev. Astron. Astrophys. 42, 275.

Schekochinin, A. A., Cowley, S. C., Dorland, W., Hammett, G. W., Howes, G. G., QuATAERT, E. \& TATsuno, T. 2009 Astrophysical gyrokinetics: kinetic and fluid turbulent cascades in magnetized weakly collisional plasmas. Astrophys. J. Suppl. 182, 310.

Schekochinin, A. A., Cowley, S. C., Kulsrud, R. M., Rosin, M. S. \& Heinemann, T. 2008 Nonlinear growth of firehose and mirror fluctuations in astrophysical plasmas. Phys. Rev. Lett. 100, 081301.

Schekochihin, A. A., Parker, J. T., Highcock, E. G., Dellar, P. J., Dorland, W. \& Hammetт, G. W. 2016 Phase mixing versus nonlinear advection in drift-kinetic plasma turbulence. J. Plasma Phys. 82, 905820212.

Shebalin, J. V., Matthaeus, W. H. \& Montgomery, D. 1983 Anisotropy in MHD turbulence due to a mean magnetic field. J. Plasma Phys. 29, 525.

Smith, C. W., VAsquez, B. J. \& HollweG, J. V. 2012 Observational constraints on the role of cyclotron damping and kinetic Alfvén waves in the solar wind. Astrophys. J. 745, 8.

Sorriso-Valvo, L., Carbone, F., Leonardis, E., Chen, C. H. K., Šafránková, J. \& NĚMEČEK, Z. 2016 Multifractal analysis of high resolution solar wind proton density measurements. Adv. Space Res. (submitted).

Sorriso-Valvo, L., Marino, R., Lijoi, L., Perri, S. \& Carbone, V. 2015 Self-consistent castaing distribution of solar wind turbulent fluctuations. Astrophys. J. 807, 86.

Stawarz, J. E., Ergun, R. E. \& Goodrich, K. A. 2015 Generation of high-frequency electric field activity by turbulence in the Earth's magnetotail. J. Geophys. Res. 120, 1845.

Sundkvist, D., Retinò, A., Vaivads, A. \& Bale, S. D. 2007 Dissipation in turbulent plasma due to reconnection in thin current sheets. Phys. Rev. Lett. 99, 025004.

TenBarge, J. M. \& Howes, G. G. 2013 Current sheets and collisionless damping in kinetic plasma turbulence. Astrophys. J. 771, L27.

Tenbarge, J. M., Podesta, J. J., Klein, K. G. \& Howes, G. G. 2012 Interpreting magnetic variance anisotropy measurements in the solar wind. Astrophys. J. 753, 107.

Šafránková, J., NěmeČek, Z., Němec, F., Přech, L., Chen, C. H. K. \& Zastenker, G. N. 2016 Power spectral density of fluctuations of bulk and thermal speeds in the solar wind. Astrophys. J. 825, 121.

Šafránková, J., Němeček, Z., Němec, F., Přech, L., Pitňa, A., Chen, C. H. K. \& ZASTENKER, G. N. 2015 Solar wind density spectra around the ion spectral break. Astrophys. J. 803, 107.

ŠAfránkovÁ, J., NĚMeČEK, Z., PŘEch, L. \& ZAStenker, G. N. 2013 Ion kinetic scale in the solar wind observed. Phys. Rev. Lett. 110, 025004. 
Vaivads, A., Retino, A., Soucek, J., Khotyaintsev, Y., Valentini, F., Escoubet, C. P., Alexandrova, O., Andre, M., Bale, S. S., Balikhin, M. et al. 2016 Turbulence heating ObserveR - satellite mission proposal. J. Plasma Phys. 82, 905820501.

VAǏnShtEľn, S. I. 1973 Strong plasma turbulence at helicon frequencies. Sov. Phys.-JETP 37, 73.

Vech, D. \& Chen, C. H. K. 2016 Testing the effects of expansion on solar wind turbulence. Astrophys. J. Lett. 832, L16.

Velli, M., Grappin, R. \& Mangeney, A. 1989 Turbulent cascade of incompressible unidirectional Alfven waves in the interplanetary medium. Phys. Rev. Lett. 63, 1807.

VERdini, A. \& GRAPPIN, R. 2015 Imprints of expansion on the local anisotropy of solar wind turbulence. Astrophys. J. 808, L34.

Verdini, A., Grappin, R., Pinto, R. \& Velli, M. 2012 On the origin of the $1 / f$ spectrum in the solar wind magnetic field. Astrophys. J. 750, L33.

Wan, M., Matthaeus, W. H., Karimabadi, H., Roytershteyn, V., Shay, M., Wu, P., Daughton, W., Loring, B. \& Chapman, S. C. 2012 Intermittent dissipation at kinetic scales in collisionless plasma turbulence. Phys. Rev. Lett. 109, 195001.

Wan, M., Matthaeus, W. H., Roytershteyn, V., Parashar, T. N., Wu, P. \& Karimabadi, H. 2016 Intermittency, coherent structures and dissipation in plasma turbulence. Phys. Plasmas 23, 042307.

WAng, X., Tu, C., Marsch, E., He, J. \& WANG, L. 2016 Scale-dependent normalized amplitude and weak spectral anisotropy of magnetic field fluctuations in the solar wind turbulence. Astrophys. J. 816, 15 .

Wicks, R. T., Horbury, T. S., Chen, C. H. K. \& Schekochinin, A. A. 2010 Power and spectral index anisotropy of the entire inertial range of turbulence in the fast solar wind. Mon. Not. R. Astron. Soc. 407, L31.

Wicks, R. T., Horbury, T. S., Chen, C. H. K. \& Schekochinin, A. A. 2011 Anisotropy of imbalanced Alfvénic turbulence in fast solar wind. Phys. Rev. Lett. 106, 045001.

Wicks, R. T., Mallet, A., Horbury, T. S., Chen, C. H. K., Schekochihin, A. A. \& Mitchell, J. J. 2013 Alignment and scaling of large-scale fluctuations in the solar wind. Phys. Rev. Lett. 110, 025003.

Yan, L., He, J., Zhang, L., Tu, C., Marsch, E., Chen, C. H. K., Wang, X., Wang, L. \& WICKS, R. T. 2016 Spectral anisotropy of Elsässer variables in two-dimensional wave-vector space as observed in the fast solar wind turbulence. Astrophys. J. 816, L24.

Zhdankin, V., Boldyrev, S. \& Chen, C. H. K. $2016 a$ Intermittency of energy dissipation in Alfvénic turbulence. Mon. Not. R. Astron. Soc. 457, L69.

Zhdankin, V., BoldyreV, S. \& MAson, J. 2012 Distribution of magnetic discontinuities in the solar wind and in MHD turbulence. Astrophys. J. 760, L22.

Zhdankin, V., Boldyrev, S., Perez, J. C. \& Tobias, S. M. 2014 Energy dissipation in magnetohydrodynamic turbulence: coherent structures or 'nanoflares'? Astrophys. J. 795, 127.

Zhdankin, V., Boldyrev, S. \& UzDensky, D. A. $2016 b$ Scalings of intermittent structures in magnetohydrodynamic turbulence. Phys. Plasmas 23, 055705.

Zhuravleva, I., Churazov, E., Schekochinin, A. A., Allen, S. W., Arevalo, P., Fabian, A. C., Forman, W. R., Sanders, J. S., Simionescu, A., Sunyaev, R. et al. 2014 Turbulent heating in galaxy clusters brightest in X-rays. Nature 515, 85.

\section{Christopher Chen}

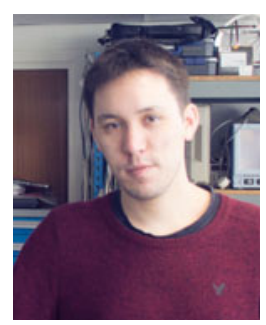

Christopher Chen is a Research Fellow at Imperial College London, where he investigates fundamental processes in space plasmas. In particular, he specialises in the use of spacecraft observations to study turbulence, instabilities and kinetic plasma physics in the solar wind. He obtained his $\mathrm{PhD}$ in 2011 at Imperial, working on data from the multi-spacecraft ESA Cluster mission. He then took up a postdoc position at the Space Sciences Lab, UC Berkeley, where he worked on Wind and ARTEMIS data and joined the NASA Solar Probe Plus mission as a member of the FIELDS team. In 2013 he returned 
to Imperial to begin a Junior Research Fellowship, and in 2016 became an Ernest Rutherford Research Fellow. He is currently a member of the Science Study Team for the ESA THOR (Turbulence Heating ObserveR) mission, as well as a Co-I of the THOR magnetometer, and serves on the executive committee of the APS Topical Group in Plasma Astrophysics. 